\title{
The role of target closure in detachment in Magnum-PSI
}

G.R.A. Akkermans*, I.G.J. Classen, H.J. van der Meiden, J. van den Berg-Stolp, and J.W.M. Vernimmen

DIFFER Dutch Institute for Fundamental Energy Research, De Zaale 20, 5612AJ, Eindhoven, the Netherlands ${ }^{*}$ Corresponding author: g.r.a.akkermans@differ.nl

\section{Abstract}

A cylindrical target with a high degree of closure was exposed to ITER divertor-relevant plasmas with typical electron temperatures of $2 \mathrm{eV}$, electron densities of $5 \cdot 10^{20} \mathrm{~m}^{-3}$, and heat fluxes up to 20 $\mathrm{MWm}^{-2}$ in the linear device Magnum-PSI. By terminating the plasma in an unpumped closed volume, neutral pressures were enhanced from about $0.5 \mathrm{~Pa}$ to $20 \mathrm{~Pa}$ without any increase in the neutral flux returning to the plasma. Such pressures were sustained largely by the pressure exerted by the incoming plasma. By means of hydrogen gas injection, internal neutral pressures of up to $40 \mathrm{~Pa}$ were reached during plasma exposure. We find that at these high neutral pressures, $\mathrm{a}<1 \mathrm{eV}$ recombination front forms and expands from the back of the cylinder, so that downstream density drops dramatically. Furthermore, in these scenarios heat deposition to the back plate vanishes and is redirected to the upstream part of the cylinder and to hot neutrals which can carry $50 \%$ of the plasma input power. A power balance analysis reveals that even without additional gas puffing, only about $10 \%$ of the incoming heat load reaches the back plate for the $20 \mathrm{MWm}^{-2}$ plasma. These results demonstrate the important role of closed target configurations and local gas puffing in mitigating plasma heat loads, and indicates that the gained experience should be taken into account in next-generation divertor designs.

\section{1 - Introduction}

One of the greatest challenges towards commercial fusion power is the handling of heat loads to plasmafacing components (PFCs). Divertor plasma detachment may offer a solution to the heat load problem, leveraging a variety of plasma-neutral processes to produce a loss of plasma pressure along the magnetic field line and a subsequent reduction in PFC heat loads.

Many advances and proposals in divertor design are aimed at increasing neutral pressure inside the divertor volume in order to stimulate such plasma-neutral interaction, while minimizing leakage of neutrals back into the main plasma which would deteriorate upstream conditions. In most present-day tokamaks, as well as the ITER divertor design, this is accomplished by structures such as domes and baffles, which reduce the spread of neutrals from the divertor to the main chamber $[1,2,3,4]$. Additionally, tilting of the target promotes reflection of neutrals away from the main plasma, and the divertor leg itself can block neutrals from reaching the upstream plasma. The Super-X divertor configuration used in MAST-U offers strong advantages in terms of flux expansion, plasma wetted area, and connection length [5], while additionally promoting neutral closure by means of the large depth of the divertor chamber [6]. The so-called slot divertor concept [7] takes structural closure of the divertor to an extreme, and has recently been implemented in the form of a Small Angle Slot divertor on DIII$\mathrm{D}$ [8]. The lithium vapor box design by Goldston et al. combines extreme structural closure with differential pumping and a downstream source of gaseous impurity in the form of a liquid lithium target [9].

Over the years, many studies of the effect of closed geometries on fusion edge plasmas have shown a beneficial effect on detachment characteristics and heat load handling [10,11, 12, 13, 14, 15, 16, 17]. The favorable effect of closure has also been observed in linear devices, for example in the tandem mirror device Gamma 10 [18], where a V-shaped target is used to accumulate neutral pressure. 
In this work, we investigate the role of closed target geometries on detachment in the linear device Magnum-PSI, which simulates plasma conditions in the lower divertor region of a (semi-)detached tokamak plasma. Hydrogen plasmas with relatively low electron temperatures $<5 \mathrm{eV}$ are produced, but nonetheless the high particle fluxes lead to large heat loads in the form of surface deposition of recombination energy. Four different cylindrical target configurations are used, having different levels of closure and diagnostic access. The principle is to terminate the plasma in a closed, unpumped subvolume, with an opening just large enough to admit the plasma and to accumulate neutral pressure, for example from recycling neutrals, inside this sub-volume. As such, the configuration with the highest degree of closure is of primary interest, while the more open configurations can provide a proxy for the (inaccessible) conditions inside the closed geometry at matching neutral pressures.

Other geometrical aspects relevant to tokamak divertor operation, such as flux expansion, higher-order magnetic nulls, drift flow patterns, and poloidal leg length $[19,20]$ are not addressed in this work. The simple linear geometry of Magnum-PSI precludes this, and instead offers the opportunity to investigate in detail the effect of structural closure on neutral pressures, and the resulting fundamental processes leading to heat load mitigation. Compared to the Gamma 10 study, this work investigates a variety of degrees of closure, and concerns plasmas of higher density and lower temperature. Hydrogen gas puffing inside the unpumped volume provided by the closed target is used to further increase internal neutral pressures for additional experimental flexibility.

This paper is structured as follows: Section 2 details the set-up of the linear device Magnum-PSI and design of the cylindrical target. Section 3.1 gives an overview of the pressures that are achieved in the different target configurations, showing that internal neutral pressures are sustained by the incoming plasma pressure. Section 3.2 constructs a power balance for the plasma heat deposition, showing that high neutral pressures lead to a reduction of heat deposition to both the back plate and the mantle of the target, such that hot neutrals must carry away a significant part of the plasma heat flux. In Section 3.3, measurements inside the cylindrical target are compared to the observed heat flux redistribution, showing the emergence of a recombination front that moves upstream with higher neutral pressure, directing the heat deposition profile. Section 4 presents the main conclusions of this work.

\section{2- Experimental set-up}

The linear device Magnum-PSI [21] produces plasma by means of a cascaded arc source, and confines this plasma in an axial magnetic field of up to $2.5 \mathrm{~T}$ produced by a superconducting $\mathrm{NbTi}$ magnet. The plasma is transported through three chambers, which are differentially pumped to remove neutrals, minimizing both plasma losses and neutral influx to the target chamber. The total distance between source and target is $1.0 \mathrm{~m}$. An overview of the device is given in Figure 1.

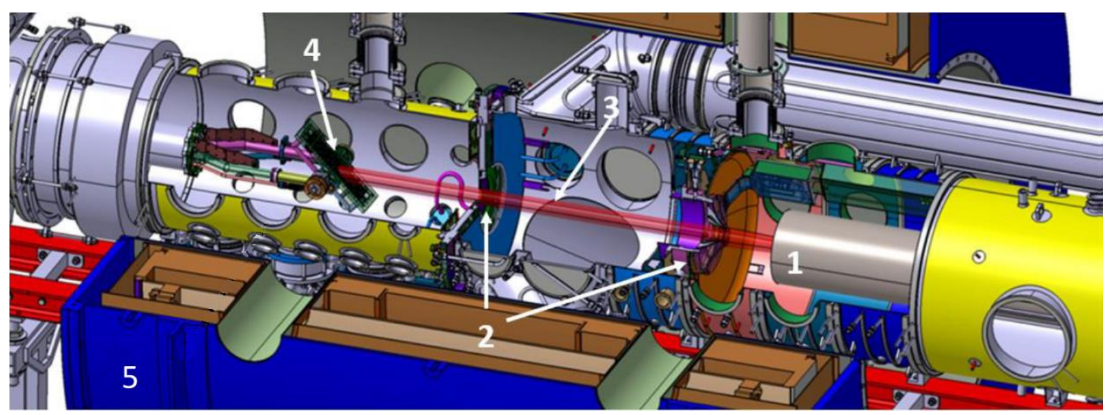

Figure 1: Design of the linear plasma device Magnum-PSI. Indicated are the plasma source (1), skimmers separating the three chambers (2), the plasma beam (3), an example target (4), and the superconducting magnet (5). 
Figure 2 shows the design of the structurally closed target of cylindrical shape that is used in this work, and the four different configurations in which it is utilized in this work. The target consists of a circular back plate made of titanium-zirconium-molybdenum (TZM) alloy with a diameter of $5 \mathrm{~cm}$, attached to one or more modules of a $2 \mathrm{~mm}$ thick, hollow cylinder made of stainless steel. It contains an inlet for hydrogen gas puffing, as well as a protrusion to which a baratron pressure gauge of type MKS 627B is connected. Holes are present in the cylinder mantle in order to allow diagnostic access with the Thomson Scattering (TS) and Optical Emission Spectroscopy (OES) diagnostics. TS measures profiles of electron temperature $T_{e}$ and density $n_{e}$ with a radial resolution of $1.3 \mathrm{~mm}$ [22]. A laser entry, laser exit, view entry and view dump allow measurements of radial profiles $25 \mathrm{~mm}$ wide at three specific axial locations along the cylinder. OES measures the intensity of the Balmer series from $H_{\beta}(\mathrm{n}=4-2)$ to the continuum ( $\mathrm{n}=\infty-2)$ with an axial resolution of $1.1 \mathrm{~mm}$ [23]. The OES view slit is $8 \mathrm{~cm}$ long, allowing a continuous axial view along the length of the cylinder. This view is integrated over a line that passes through the OES entrance slit and the axis of the cylinder.

14

15 a) $10 \mathrm{~cm}$, open

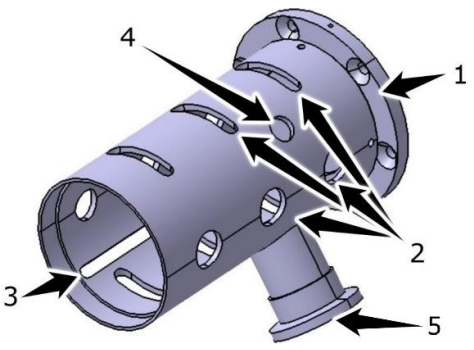

c) $10 \mathrm{~cm}$, closed

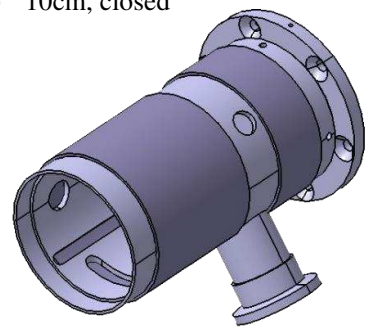

b) $10 \mathrm{~cm}$, semi-open
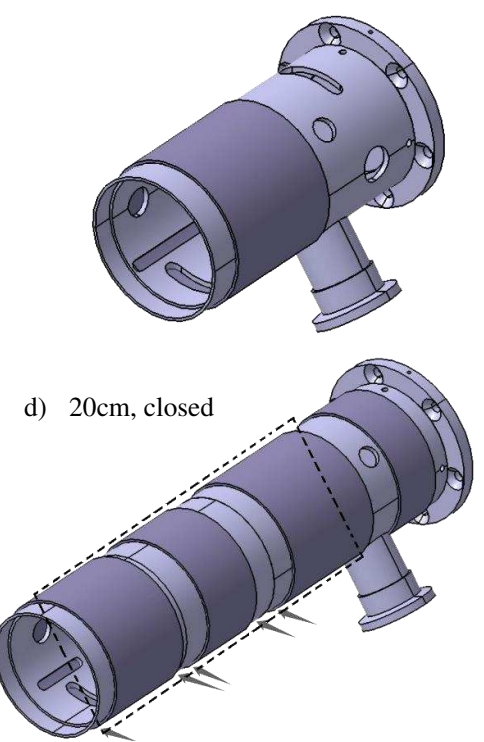

Figure 2: Target configurations used in this work. a: $10 \mathrm{~cm}$, open target configuration, consisting of a circular TZM back plate of $5 \mathrm{~cm}$ diameter (1), attached to a $10 \mathrm{~cm}$ long stainless steel cylinder. Thomson scattering utilizes three sets of 4 open diagnostic ports (2). Spectroscopy uses a single $8 \mathrm{~cm}$ long slit (3). The locations of gas inlet (4) and the baratron gauge (5) are also marked. b: $10 \mathrm{~cm}$, semi-closed configuration, with a stainless steel foil covering the front of the cylinder. c: $10 \mathrm{~cm}$, closed configuration, with an additional foil covering the back of the cylinder. d: $20 \mathrm{~cm}$, closed configuration, where two $5 \mathrm{~cm}$ long cylinder modules are attached, both with stainless steel cover. The locations used for pyrometer measurements are marked by the grey arrows, and the view of the monitoring camera by the black box. 
Table 1: Overview of the plasma scenarios studied. The chosen magnetic field $B$, source current $I_{s}$, and gas flow $\Gamma_{s}$ are reported, as well as the main results of Thomson Scattering, measured $5 \mathrm{~mm}$ in front of the cylinder opening: peak electron temperature, density, and FWHM of the density profile.

\begin{tabular}{|l|l|l|l|l|l|}
\hline$I_{S}[\mathrm{~A}]$ & $B[\mathrm{~T}]$ & $\Gamma_{S}[\mathrm{slm}]$ & $T_{e, \text { peak }}[\mathrm{eV}]$ & $n_{e, \text { peak }}\left[10^{20} \mathrm{~m}^{-3}\right]$ & $n_{e}$ FWHM $[\mathrm{mm}]$ \\
\hline 140 & 1.0 & 6.0 & $2.3 \pm 0.2$ & $1.43 \pm 0.04$ & 14 \\
\hline 170 & 1.0 & 5.0 & $1.5 \pm 0.1$ & $5.0 \pm 0.2$ & 14 \\
\hline
\end{tabular}

In some target configurations, the TS and OES holes are covered by a stainless steel foil to provide additional closure at the cost of diagnostic access. The different target configurations that are used are: $10 \mathrm{~cm}$, open (Figure 2a);10 cm, semi-closed (Figure 2b);10 cm, closed (Figure 2c); $20 \mathrm{~cm}$, closed (Figure 2d). Note that holes 4 and 5 in Figure 2 are always closed due to the connections with the gas source and baratron. Different levels of gas puff are required to reach the same pressure in the various target configurations, making plasma parameters measured in the more open configurations a suitable proxy for the conditions inside the closed target geometries, where diagnostic access is blocked, at corresponding neutral pressures.

Additionally, thermocouples are present in the back plate and on the back end of the cylinder mantle. The back plate thermocouple, specifically, is calibrated to the standard calorimetry system that infers target heat loads using the temperature increase of the cooling water, showing agreement to within $90 \%$. Finally, a pyrometer is used to obtain an emissivity-independent measurement of the surface temperature of different parts of the cylinder mantle. The measurement position is flexible, by means of linear movement of the target. The system consists of a lens assembly and a FAR-Associate SpectroPyrometer of model FMPI.

The various configurations of closed cylindrical target have been exposed to two different quasi-steady state hydrogen plasma scenarios in Magnum-PSI. These are produced by a set of machine settings consisting of the axial magnetic field $B$, the plasma source current $I_{s}$, and the hydrogen gas feed $\Gamma_{s}$, for which the unit standard liters per minute $(\mathrm{slm})$ is used $\left(1 \mathrm{slm}=4.4 \cdot 10^{20} \mathrm{particles} / \mathrm{s}\right)$. The chosen machine settings and resulting plasma parameters are listed in Table 1 . The listed uncertainties are the $1 \sigma$ standard deviations of repeated measurements with 90 laser pulses, mainly limited by the stability of the plasma source.

\section{3 - Experiments on closed target geometries in Magnum-PSI}

The main result of this work is the study of how plasma heat flux is distributed for different levels of neutral pressure in the most closed target configuration. A first impression of the behavior of heat distribution is provided by the in-vessel monitoring camera. During plasma exposures of several minutes, the mantle of the cylindrical target heats up far enough that it starts to glow, and this blackbody radiation is recorded by the monitoring camera. The viewing geometry of the camera was previously indicated in Figure 2d.

Figure 3 shows a series of such camera images for the $20 \mathrm{~cm}$, closed target configuration. In the case of no internal gas puff, black-body radiation from the cylinder itself is concentrated around the center, as seen in Figure 3a. Figure 3b and c show that as gas puff is applied and increased, the black-body radiation shifts towards the front of the cylinder, i.e. in the upstream direction. It should be noted that the stainless steel foils covering most of the diagnostic ports emit much less than the bare parts of the cylinder, likely through a combination of low emissivity and poor thermal contact with the cylinder beneath.

There are limitations to inferring perpendicular heat flux distribution from plain camera images of the cylinder mantle's black-body emissions. Conduction along the mantle tends to smooth out emissions, heat deposited by previous plasma exposures may still contribute to emmission profiles, and poor 
thermal contact between the different modules causes discontinuities in observed emissions. Perhaps most importantly, the monitoring camera does not resolve the emissions spectrally, so that it cannot quantify target temperatures and heat fluxes. Nonetheless, the strong localization of black-body emission for cases with high gas puff implies a significant redistribution of heat flux away from the back plate. This redistribution of heat flux is studied quantitatively using pyrometer measurements in section 3.2 .

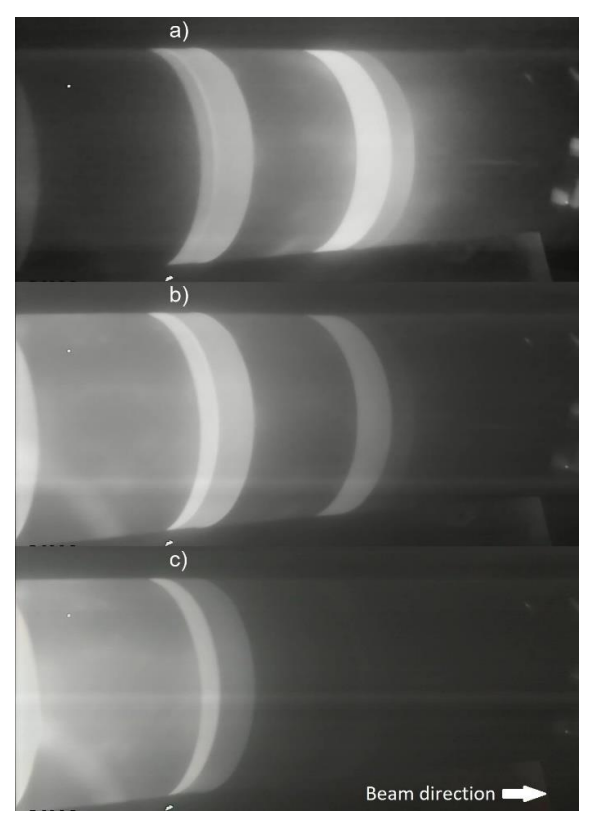

Figure 3: Monitoring camera images of the infra-red afterglow of the $20 \mathrm{~cm}$, closed cylindrical target after exposure to a 170 A Magnum-PSI plasma in case of 0 (a), 3 (b) and 5 (c) slm of gas puff. The presence of gas puff causes significant redistribution of perpendicular plasma heat flux.

\section{1 - Pressure accumulation inside closed target geometries}

This section gives an account of the neutral pressures reached inside the cylindrical target, showing that the cylinder is 'plugged' by the force of the plasma at its entrance. An overview of the obtained neutral pressures is given in Figure 4, where the internal neutral pressure measured by the baratron gauge is plotted as a function of gas puff level, for different target configurations and plasma source settings. Internal neutral pressures of up to $40 \mathrm{~Pa}$ are reached.

The neutral pressure generally increases as a function of increased source current, target closure, and hydrogen gas puff level. The behavior as a function of gas puff and target closure is clear; an increase in the source of neutral particles or their level of trapping leads to more accumulation of neutrals inside. At high levels of gas puff, the enhancement of neutral pressure stalls or even reverses. This will be explained in more detail after a treatment of the behavior of neutral pressure as a function of plasma source current.

The strong increase in neutral pressure with plasma settings can only partially be explained by an increased source of neutrals due to recycling. The recycling source is determined from the neutral pressure measured in the vacuum vessel surrounding the cylindrical target, which is sensitive to a 
release of either recycled or puffed neutrals from the target. The recycling source of neutrals for the 170 A plasma is thus found to be $0.95 \mathrm{slm}$. If recycling neutrals in cases with plasma are trapped only by the structure of the target, at the same effectiveness as puffed neutrals in cases without plasma, then 1 slm of gas puff with no plasma should result in a similar neutral pressure as the 170 A plasma with no gas puff. However, comparing these two cases for the $20 \mathrm{~cm}$ closed target configuration reveals that in the case with plasma, neutral pressure is a factor 3 higher. This difference must be due to the plasma 'plugging' the upstream end of the cylinder, exerting pressure on the neutrals and preventing their escape through the front opening.

The plasma plugging effect is stronger than would be expected from a simple gas conductance perspective. In order to overestimate the effect of the 'wings' in the electron density and temperature profiles, we can approximate the density profile as a Gaussian distribution with standard deviation $\sigma=$ $6 \mathrm{~mm}$ and take $2 \sigma$ as the overestimated plasma radius. In this overestimated case, the plasma still only covers $23 \%$ of the area of the cylinder entrance. This is significantly short of the $67 \%$ that would be required to produce a factor 3 increase in neutral pressure. Clearly, the plasma does more than seal off part of the entrance to the cylinder, like a metal wall would. It applies an additional active force to the gas inside.

The eventual decrease of internal neutral pressure at high levels of gas puff can be explained by the same plugging effect. The moderate pressure build-up of up to $2 \mathrm{~Pa}$ outside the target likely causes neutrals to interact with the plasma before it reaches the cylinder entrance, resulting in lower incoming plasma pressure and a weaker plugging effect.

Figure 5 compares the neutral pressure inside the target to the peak incoming static plasma pressure. The decrease of plasma pressure by typically a factor two over the measured gas puff range confirms that the incoming plasma is significantly affected by the gas puffed inside the target. For most plasma settings and target geometries, a local peak in internal neutral pressure occurs when the neutral pressure reaches about $30 \%$ of the plasma pressure. This marks the point where the reduced effectiveness of plasma plugging due to the lower plasma pressure outweighs the extra source of neutrals from gas puffing. In this analysis, the static plasma pressure was calculated from Thomson Scattering measurements $5 \mathrm{~cm}$ in front of the cylinder opening as $2 k_{B} n_{e} T_{e}$ with $k_{B}$ the Boltzmann constant, i.e. assuming ion-electron temperature equilibration.

30

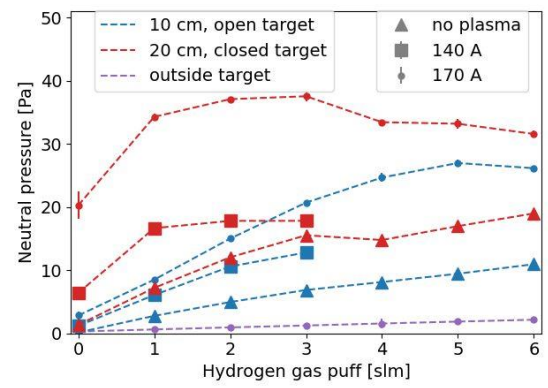

Figure 4: Neutral pressure inside target from baratron gauge as function of gas puff rate for different plasma source settings (symbols) and target configurations (color). The data in purple is the neutral pressure outside the target, also for the $20 \mathrm{~cm}$, closed target configuration. Neutral pressure increases with target closure and with gas puff level due to plasma plugging, but eventually stalls or even decreases again at high levels of gas puff. 


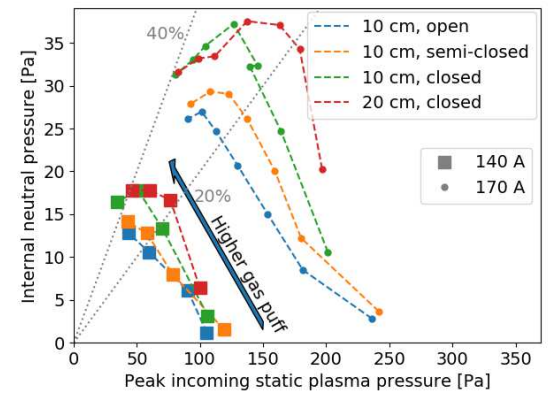

Figure 5: Comparison of peak static plasma pressure from Thomson Scattering $5 \mathrm{~cm}$ in front of the cylinder opening with neutral pressure inside the cylinder, for different target configurations (colors) and plasma settings (symbols). The level of gas puff varies along the curves (steps of $1 \mathrm{slm}$ starting at $0 \mathrm{slm}$, arrow indicates direction). Neutral pressure peaks when it reaches $\sim 30 \%$ of the incoming plasma pressure.

Given the explanation of plasma plugging, it might be expected that the neutral pressure should be able to reach $100 \%$ of the plasma pressure rather than $30 \%$. However, the plasma beam (with $n_{e}$ FWHM of $14 \mathrm{~mm}$ for the plasmas studied here) is much thinner than the cylindrical target (diameter $50 \mathrm{~mm}$ ), such that the plasma can only effectively plug the center of the target entrance and neutrals can still escape from the edge region of the entrance. A thinner cylinder to tightly fit the plasma beam would likely reach a higher ratio of neutral to plasma pressure.

It is worthwhile to note that the neutral pressure enhancement due to target closure is achieved without any effect on the neutral flux returning to the plasma, and hence the upstream plasma parameters. This neutral influx is determined by the sum of recycling and gas puff and is independent of the residence time or internal pressure of the neutral particles, under the assumption that all particles leave the cylinder as neutrals.

In summary, the results in this section have shown that in Magnum-PSI, closed target geometries lead to significant accumulation of internal neutral pressure, which is 'plugged' by the pressure of the incoming plasma.

\section{2 - Redistribution of heat flux at high neutral pressures}

In this section, we address how the accumulation of neutral pressure affects the spatial distribution of the heat load presented by the plasma, showing that the abundance of neutrals shifts heat loads to the front of the cylinder and causes a significant fraction of the heat to escape in the form of hot neutrals, rather than reach the target structure. Target heat loads are measured in several locations, and a power balance is used to investigate whether these measurements account for the full plasma heat load. This power balance is constructed as follows:

$$
Q_{\text {in }}=Q_{\text {back }}+Q_{\text {sides }}+Q_{\text {gas }}
$$

where $Q_{i n}$ is the incoming plasma heat load measured by Thomson Scattering, $Q_{\text {back }}$ is the parallel heat load to the back plate measured by a thermocouple, $Q_{\text {sides }}$ is the perpendicular heat load to the mantle of the cylinder measured by the pyrometer, and $Q_{\text {gas }}$ is residual heat escaping in the form of hot neutrals.

$Q_{\text {in }}$ is calculated from TS profiles measured $5 \mathrm{~mm}$ in front of the entrance of the cylindrical target. Heat flow into the cylindrical target is assumed to be convective, and in the absence of a measurement of the 
plasma flow velocity, a Mach number of 0.5 is assumed. Furthermore, it is assumed that $T_{e}=T_{i}$. The heat flux density $q_{\|, \text {in }}$ entering the cylinder is then given by [25]:

$$
q_{\|, \text {in }}=0.5 n_{e} c_{s}\left(5 k_{B} T_{e}+\chi_{i}+\chi_{r}\right) \text {, }
$$

where $c_{S}=\left(\frac{(1+\gamma) k_{B} T_{e}}{m_{i}}\right)^{1 / 2}$ is the plasma sound speed for adiabatic flow $(\gamma=5 / 3)$ and $\chi_{i}=$ $13.5 \mathrm{eV}$ and $\chi_{r}=2.2 \mathrm{eV}$ are the ionization and dissociation energies of hydrogen, respectively.

$Q_{b a c k}$ is obtained from the thermocouple installed in the back plate, and can be found from the jump in the time-derivative $\Delta\left(\frac{\mathrm{d} T}{d t}\right)$ of the recorded temperature at the moment the plasma exposure is initiated:

$$
Q_{\text {back }}=m c_{p} \Delta\left(\frac{\mathrm{d} T}{d t}\right)=\pi R^{2} q_{\| \cdot}
$$

Here, $m=0.245 \mathrm{~kg}, c_{p}=305 \mathrm{Jkg}^{-1} \mathrm{~K}^{-1}$, and $R=50 \mathrm{~mm}$ are the mass, specific heat capacity, and radius of the TZM back plate, respectively. $Q_{b a c k}$ is also rewritten in terms of the average parallel heat flux $q_{\|}$reaching the back plate.

An overview of the behavior of $q_{\|}$is shown in Figure 6, showing a great reduction in parallel heat flux at high levels of gas puff, i.e., high neutral pressures. From Figure 6a, it is clear that $q_{\|}$decreases with gas puffing for all target configurations and both plasma settings. When $q_{\|}$is plotted as a function of neutral pressure inside the target, as in Figure $6 \mathrm{~b}$, the results for different target configurations under the same plasma settings fall broadly on the same curve. This shows that internal neutral pressure, determined by the balance of target geometry and gas injection, is the governing parameter for the mitigation of the plasma heat flux.

a)

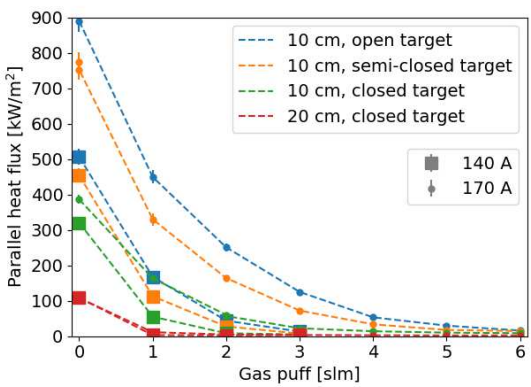

b)

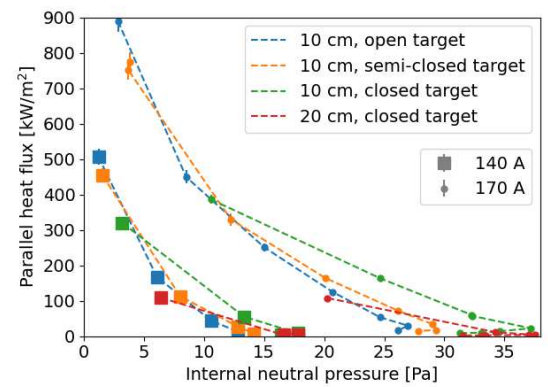

Figure 6: Parallel heat flux from thermocouple as a function of (a) level of gas puff or (b) internal neutral pressure, for different plasma settings (symbols) and target configuration (colors). Neutral pressure, rather than gas puff alone, is the governing parameter for the behavior of heat flux.

$Q_{\text {sides }}$ is found from pyrometer measurements on the outer mantle of the cylinder. Perpendicular heat fluxes $q_{\perp}$ are extracted from the observed jump in time derivative of temperature, equivalent to how $q_{\|}$ is determined from Eq. (3). Here, $c_{p}=500 \mathrm{Jkg}^{-1} \mathrm{~K}^{-1}$ and an areal mass density of $14.2 \mathrm{~kg} / \mathrm{m}^{2}$ is used. In order to obtain axial profiles of $q_{\perp}$, the position of the cylindrical target as a whole is shifted so that the pyrometer is focused on the different positions indicated in Figure 2d. The thermocouple in the back of the cylinder mantle yields an additional measurement position for $q_{\perp}$. Since the neutral pressures inside the target are much larger than those outside, the aforementioned shifts in target position have relatively little influence on the plasma parameters at the opening of the cylinder, and the 
conditions inside the cylinder are assumed to remain the same. Baratron and thermocouple readings for these shifted positions are constant within $20 \%$.

Axial profiles of $q_{\perp}$ are shown for both plasma settings for the $20 \mathrm{~cm}$, closed target configuration in Figure 7, showing that accumulation of neutral pressure causes perpendicular heat deposition to shift upstream, as well as gradually decrease as a whole. In the cases without gas puffing, perpendicular heat deposition is focused in the center of the cylinder in both the 140 and 170 A plasma scenarios, more strongly so for the $170 \mathrm{~A}$ plasma. The thermocouple measurement at the back of the mantle, in particular, shows a heat flux in the absence of gas puff that is a factor of 3-4 lower than the peak $q_{\perp}$ measured at the center of the mantle, suggesting significant heat load mitigation even without an external source of neutrals. At the high levels of gas puff, the strong upstream shift of the heat deposition profiles suggests an almost complete recombination and heat transfer to neutrals in the first few $\mathrm{cm}$ inside the target.
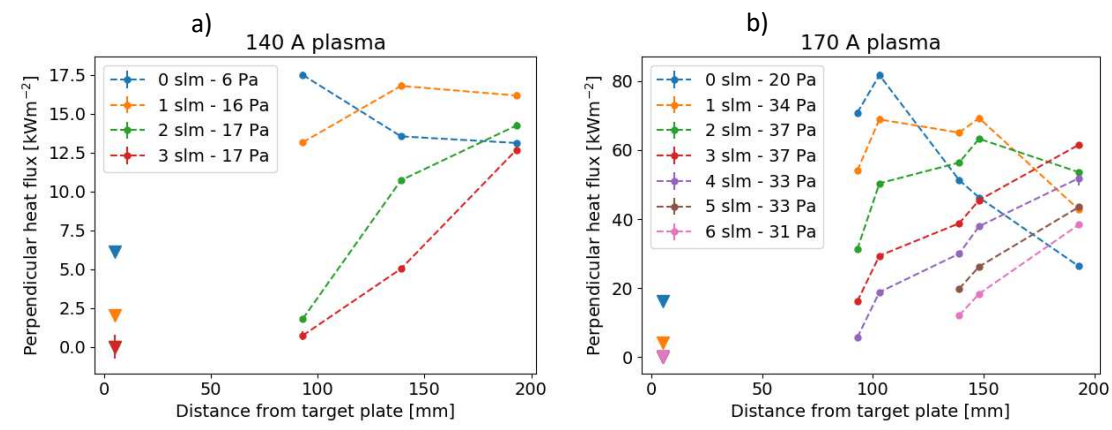

Figure 7: Axial distribution of perpendicular heat flux along the mantle of the $20 \mathrm{~cm}$, closed cylindrical target from pyrometer data (dots and dashed lines) and from the thermocouple at the back of the cylindrical mantle (triangles), for different gas seeding rates (colors, steps of $1 \mathrm{slm}$ starting at $0 \mathrm{slm}$ ) for a $140 \mathrm{~A} \mathrm{(a)} \mathrm{and} 170 \mathrm{~A}$ (b) plasma. As gas puff is increased, perpendicular heat flux shifts towards the front of the cylinder, and decreases as a whole. Note that some triangle data points are overlapping; in both cases, the thermocouple registers a vanishing heat flux for puff rates of $2 \mathrm{slm}$ and higher.

The 170 A plasma is the only condition in which all 5 measurement positions for the pyrometer are used. By comparison to the neighboring measurements at 105 and $150 \mathrm{~mm}$, it can be seen that the measurements at 95 and $140 \mathrm{~mm}$ are systematically underestimated by about $10 \%$, which is due to a detail in the structural connection of the cylinder segments. These same measurement positions are used for the other plasma settings, where they likely underestimate local heat fluxes by a similar factor. No correction is made for this systematic error, since any correction factor would be arbitrary.

The final term in the power balance of eq. (1), $Q_{g a s}$, is heat unaccounted for by the thermocouple and pyrometer measurements, which is assumed to be carried by hot neutrals, which leave the cylinder through any of its openings instead of impacting - and transferring their energy to - part of the structure. Heat escape in the form of radiation is also possible, but as the front opening represents only $6 \%$ of the total surface area of the cylinder, most radiative heat losses are likely accounted for in the $Q_{\text {sides }}$ term. 
a)

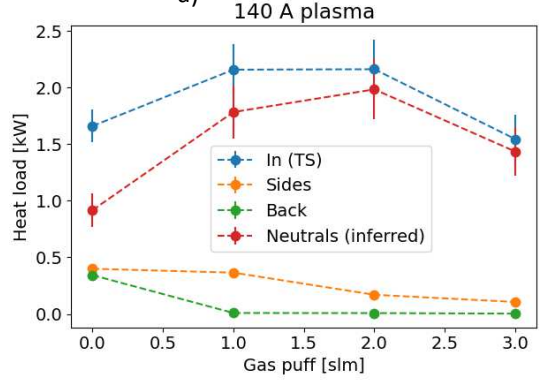

b)

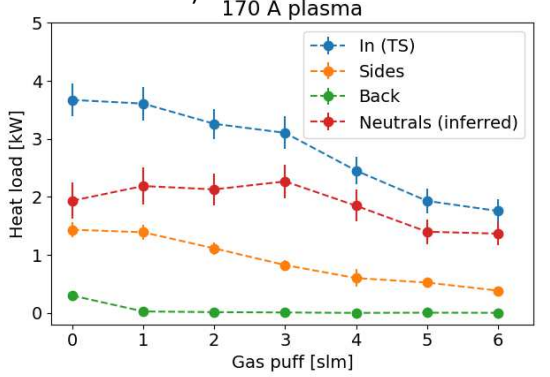

Figure 8: Power balance of heat fluxes in the cylindrical target. The total incoming heat load (Thomson Scattering), parallel heat load to the target back plate (back thermocouple), perpendicular heat load to the mantle of the target (pyrometer and side thermocouple), and outflow of hot gas (calculation) are plotted as a function of gas puff for a $140 \mathrm{~A}$ (a) and $170 \mathrm{~A}$ (b) plasma.

The plasma power distribution is shown as a function of gas puff for different plasma conditions using the $20 \mathrm{~cm}$, closed target configuration, in Figure 8. Even in cases without gas puffing, only of the incoming plasma heat flux reaches the back plate in for the $140 \mathrm{~A}$ plasma, and $8 \%$ for the $170 \mathrm{~A}$ plasma. This shows that by trapping the plasma neutrals alone, a strong degree of heat load reduction is obtained. With the application of gas puffing, $Q_{\text {back }}$ is reduced dramatically, showing complete heat load mitigation. $Q_{\text {sides }}$ also decreases as a function of gas puff rate, but not much faster than the incoming plasma load. Together, the observed $Q_{\text {back }}$ and $Q_{\text {sides }}$ account for less than $50 \%$ of the plasma heat load calculated from TS measurements. The complement, $Q_{\text {gas }}$, must escape in the form of hot neutrals, part of which may be dissociated.

One peculiarity of the data in Figure 8 is that the incoming plasma heat load, $Q_{i n}$, actually increases for the 140 A plasma, going from no gas puff to $1 \mathrm{slm}$ gas puff. This is surprising, as the neutral gas can at most provide a source of particles but not power to the plasma. Indeed, an increase in $n_{e}$ of a factor 2 and a reduction in $T_{e}$ is measured (not shown here). This suggests that the Mach number of the plasma flow decreases with gas puff, but unfortunately no measurement of the plasma flow velocity was available for these experiments.

In summary, the results of this section have shown that at high neutral pressures, heat deposition to all parts of the cylindrical target decreases and perpendicular heat deposition is shifted towards the front of the cylinder mantle. However, this shift does not account for the total plasma heat load: a significant fraction of the incoming heat is carried outside the target by escaping hot neutrals.

\section{3 - Plasma behavior inside the cylindrical target}

In order to gain additional insights into the processes inside the cylindrical target that are responsible for the observed heat load mitigation and redistribution, TS and OES measurements from the interior are used. By comparing in-target measurements in the $10 \mathrm{~cm}$, open target configuration to heat flux distributions for the $20 \mathrm{~cm}$, closed target configuration at the same neutral pressures, we find that at high neutral pressures, a recombining region is formed which drives the heat deposition profile upstream. The TS and OES measurements are taken at the 3 positions inside the cylindrical target marked in Figure 2a, as well as $5 \mathrm{~mm}$ in front of the cylinder opening. 
a)

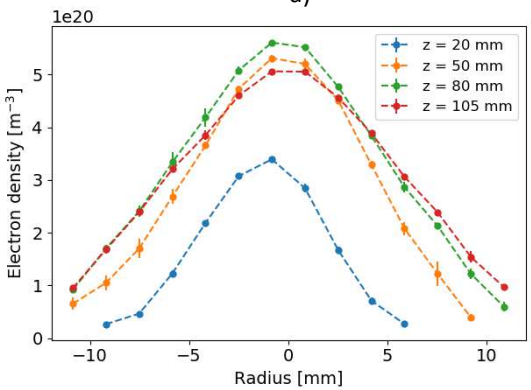

b)

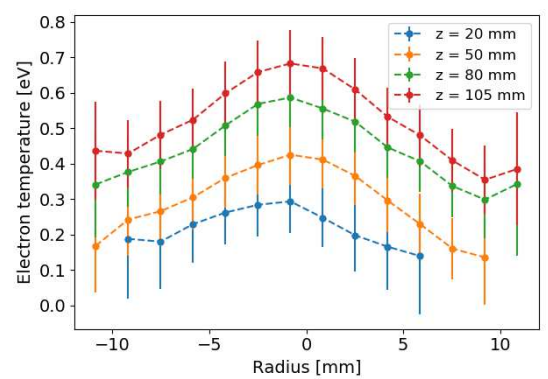

Figure 9: Radial profiles of $T_{e}$ (a) and $n_{e}$ (b) measured using Thomson Scattering at different axial positions inside and outside the $10 \mathrm{~cm}$, open target. A 170 A plasma setting with 4 slm gas puff (26.7 Pa internal pressure) is shown. $T_{e}$ decreases throughout the cylinder, while $n_{e}$ decreases strongly near the back. Profile shapes do not change strongly with axial distance.

An example of TS results for a 170 A plasma with 4 slm of gas puff is shown in Figure 9. It is shown that $T_{e}$ decreases continuously as the plasma traverses the cylinder, while the reduction in $n_{e}$ is localized near the back plate. Another observation is that the profile shapes are quite insensitive to either axial position or plasma settings. The lack of any broadening in the $n_{e}$ profile shape suggests that volume recombination, rather than radial particle diffusion, is responsible for the drop in density.

By the same reasoning, further analysis is based on the central values of radial profiles only. The resulting axial profiles of $T_{e}$ and $n_{e}$ are presented in Figure 10, along with OES results for the intensity of the Balmer- $\beta$ line. These OES measurements are integrated over a line of sight that intersects the plasma axis, and multiple fibers offer a view at multiple axial locations in a single measurement.

Figure 10a and $\mathrm{b}$ show that $T_{e}$ inside the cylindrical target decreases monotonically towards the back plate and with higher levels of gas puff in virtually all conditions. Figure $10 \mathrm{c}$ shows that $n_{e}$ globally increases as a function of gas puff at low pressures for the 140 A plasma setting, while both Figure $10 \mathrm{c}$ and $\mathrm{d}$ show that at the higher levels of gas puff, $n_{e}$ decreases strongly in the vicinity of the back plate. $H_{\beta}$ emissions, plotted in Figure 10e and $\mathrm{f}$, are localized near the back of the cylinder in the case of no gas puff. With the addition of gas puffing, $H_{\beta}$ emissions are significantly increased and shifted towards the center and front of the cylinder.

These results support the interpretation that the accumulation of neutrals inside the target leads to significant plasma-neutral energy transfer, mostly through elastic collisions such as charge exchange. These elastic collisions also cause plasma momentum losses, slowing the plasma down and leading to the observed increase in $n_{e}$ in the absence of very strong particle sinks and sources $-T_{e}<2 \mathrm{eV}$ are too low for ionization to explain the increase in density. Once $T_{e}$ is lowered to about $0.5 \mathrm{eV}$, a recombining region develops at the back plate, and gradually spreads upstream with increased gas puff. The $H_{\beta}$ emission profiles are not strongly affected by changes in the plasma parameters and seem to be dominated by the availability of neutrals: recycling atoms near the back plate in cases without gas puffing, and injected molecules in cases with gas puffing. In the case of the puffed molecules, it is likely that Molecular Activated Recombination (MAR) is the main source of radiation in most plasma scenarios. Indications that point towards this are that the $H_{\beta}$ emissions are localized near the neutral injection site, and increase in intensity with an increased source of neutrals. This increase is especially noticeable at lower puff rates for which electron temperatures are in a suitable range for MAR. In cases where the $T_{e}$ drops significantly below $1 \mathrm{eV}$, three-body recombination emission also becomes important. 
a)
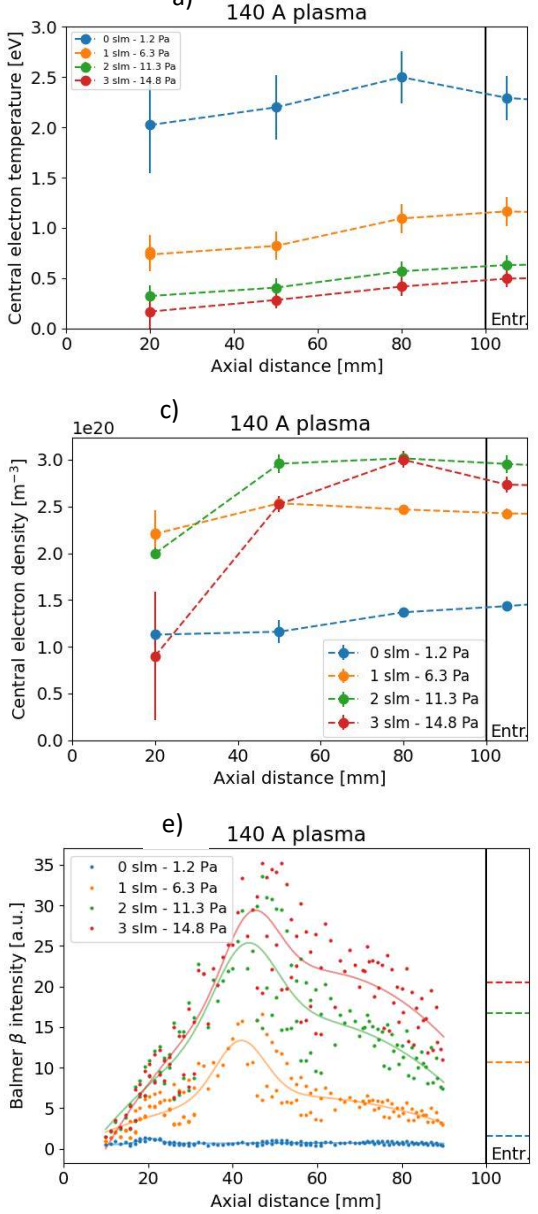

b)
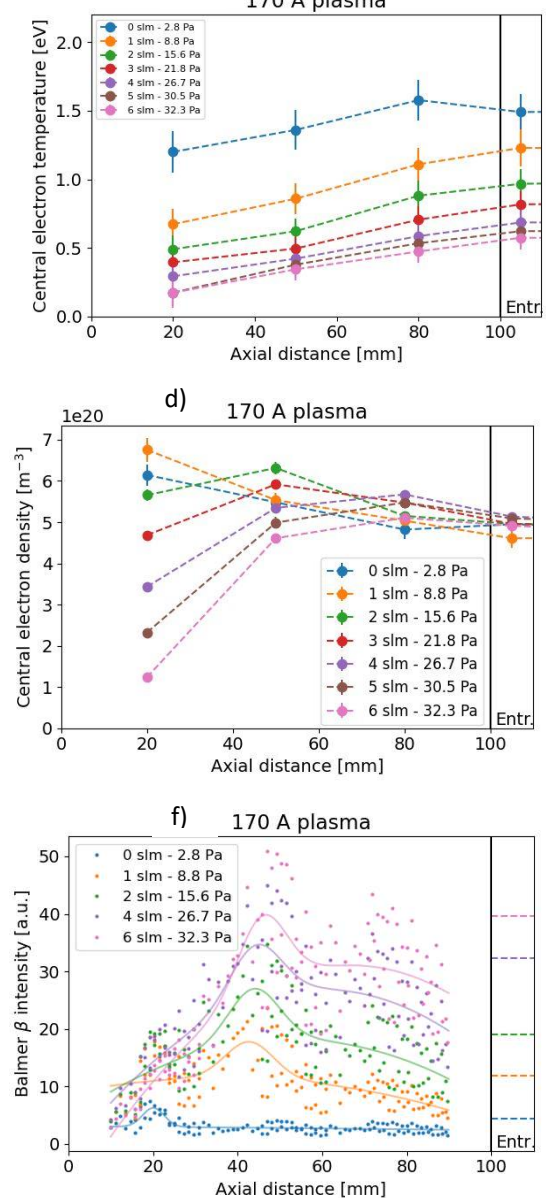

3

Figure 10: Overview of Thomson Scattering and spectroscopic results on the open target. Axial profiles of electron temperature $(\mathrm{a}, \mathrm{b})$, electron density $(\mathrm{c}, \mathrm{d})$, and the brightness of the $H_{\beta}$ line $(\mathrm{e}, \mathrm{f})$ are plotted for the $140 \mathrm{~A}(\mathrm{a}, \mathrm{c}, \mathrm{e})$ and $170 \mathrm{~A}$ (b,d,f) plasma settings, at different gas puff levels (curves, 1slm steps starting at $0 \mathrm{slm}$ ). The faded lines through the OES data are guides to the eye, the dashed lines outside are averages of all values measured outside the cylindrical target.

The presence of a recombining region can be experimentally verified by analysis of higher- $n$ Balmer lines. This is done for the 170 A plasma with $6 \mathrm{slm}$ of gas puffing in Figure 11, again in the $10 \mathrm{~cm}$, open target configuration. Line intensities of the Balmer $\beta$ line $(n=4 \rightarrow 2)$ up to $n=12 \rightarrow 2$ are extracted from the spectrum measured at $z=20 \mathrm{~mm}$, corresponding to the backmost TS measurement location. That is, Figure 11 shows data for the most deeply detached plasma scenario, measured downstream of the recombination front. The measured line intensities are converted to excited state densities $n_{q}$ and divided by the statistical weight $g_{q}$ of each excited state $q$. The normalized exited state densities $n_{q} / g_{q}$ are plotted in Figure 11. The distribution of the upper excited states is compared to a Saha distribution 
at the local electron temperature, i.e. a straight line with slope $-\frac{1}{T_{e}}$ with where $T_{e}$ is given by the local TS measurement, showing very good agreement. The lower excited states are underpopulated compared to this Saha distribution, demonstrating the dominance of electron-ion recombination in the production of excited hydrogen atoms [26]. The expected EIR reaction rate for these conditions $\left(T_{e}=0.17 \mathrm{eV}\right.$, $\left.n_{e}=1.2 \cdot 10^{20} \mathrm{~m}^{-3}\right)$ is $2.3 \cdot 10^{16} \mathrm{~m}^{3} \mathrm{~s}^{-1}$ [27], consistent with an important role for EIR.

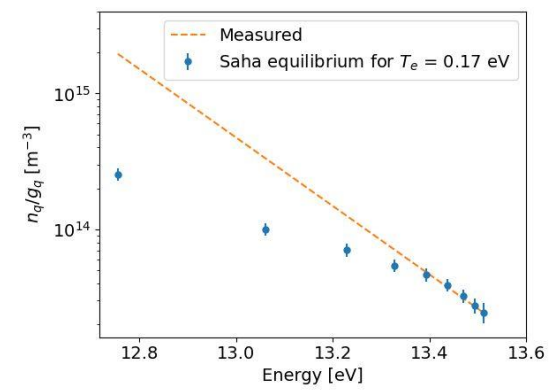

Figure 11: Population distribution of excited hydrogen atoms for the 170 A plasma with 6 slm of gas puffing in the $10 \mathrm{~cm}$, open target configuration. The recombining character of the plasma radiation is clearly shown, with the highly excited states following Saha distribution according to the local electron temperature (measured with Thomson Scattering), and the lower excited states being underpopulated relative to said distribution.

The (limited) overlap between the achieved pressure ranges for the $20 \mathrm{~cm}$, closed target configuration and the $10 \mathrm{~cm}$, open target configuration allows for some comparisons between the measurements of both parallel and perpendicular heat flux of Figure 6 and Figure 7, and the in-target measurements shown here. For example, for the 140 A plasma setting, a pressure of $6 \mathrm{~Pa}$ is reached without gas puff in the $20 \mathrm{~cm}$, closed target configuration, and approximately the same pressure is reached with $1 \mathrm{slm}$ of gas puff in the $10 \mathrm{~cm}$, open configuration. The closed configuration showed a relatively flat perpendicular heat deposition profile and significant heat load to the back plate, and the corresponding open target results show a relatively flat density and temperature profile. Around $15 \mathrm{~Pa}$, very little heat flux reached the back plate and the $q_{\perp}$ profile was shifted towards the front in the closed configuration, and the open configuration shows a sharp decrease in density near the target. In other words, the appearance of the recombination front relates to the vanishing of the heat load to the back plate, and its movement relates to the shift in perpendicular heat distribution.

\section{4 - Conclusions and outlook}

Closed target geometries were found to lead to accumulation of internal pressures up to $40 \mathrm{~Pa}$ in Magnum-PSI, with the target being 'plugged' by the incoming plasma pressure. The internal neutral pressure was shown to be the governing parameter for parallel heat flux mitigation for various combinations of target geometry and gas puff. In the configuration with the highest degree of closure in the absence of gas puff, a plasma heat load with a central heat flux density of $20 \mathrm{MWm}^{-2}$ was reduced by a factor 10 before reaching the target back plate, with $40 \%$ of the heat being deposited on the sides of the target structure as perpendicular heat, and 50\% escaping in the form of hot neutrals. In-target gas injection of at least $1 \mathrm{slm}$ causes near-complete mitigation of parallel heat loads. The reduction in parallel heat flux is accompanied by an upstream shift of the perpendicular heat deposition profile, which is related to the movement of a recombination front inside the cylindrical target.

These results suggest that in next-generation tokamaks, divertor designs with an unpumped sub-volume in the lower divertor where neutral pressures are allowed to accumulate can also play a major role in 


\begin{abstract}
mitigating heat fluxes to PFCs to tolerable levels. Upstream of this sub-volume there should be a pumped volume as in present-day tokamaks where the returning neutral flux is removed before reaching the core. Local hydrogen gas injection in the unpumped sub-volume could be used to reach even higher neutral pressures at the cost of an increased flux of neutrals to the core plasma. In a tokamak geometry, the cylindrical target shape used here translates into a narrow slit divertor. Active cooling of the sides of such a slit is necessary to handle long pulse or steady-state heat loads. The higher neutral densities and better divertor performance due to the closed target structure could be achieved without the cost of increased backflow of neutrals to the core plasma when compared to an open target geometry.
\end{abstract}

\title{
Acknowledgements
}

We acknowledge the support of the Magnum-PSI Facility Team at DIFFER. The Magnum-PSI facility at DIFFER has been funded by the Netherlands Organisation for Scientific Research (NWO) and EURATOM.

This work has been carried out within the framework of the EUROfusion Consortium and has received funding from the Euratom research and training programme 2014-2018 and 2019-2020 under grant agreement No 633053. The views and opinions expressed herein do not necessarily reflect those of the European Commission.

\section{Data Availability}

The data that support the findings of this study are available from the corresponding author upon reasonable request.

\section{Bibliography}

[1] R.A. Pitts, A. Kukushkin, A. Loarte, A. Martin, M. Merola, C.E. Kessel, V. Komarov and M. Shimada, Phys. Scr. T138 (2009) 014001 (10pp)

[2] R. Schneider, H.-S. Bosch, D. Coster, J.C. Fuchs, J. Gafert, G. Haas, A. Herrmann, M. Kaufmann, A. Kallenbach, J. Neuhauser, J. Schweinzer, U. Wenzel, and ASDEX Upgrade Teams, Journ. Nuc. Mater. 266-269 (1999) 175-181

[3] J. Rapp, W. Fundamenski, L.C. Ingesson, S. Jachmich, A. Huber, G.F. Matthews, P. Morgan, M.F. Stamp, and JET-EFDA Contributors, Plasma Phys. Control. Fusion 50 (2008) 095015 (30pp)

[4] H. Reimerdes, S. Alberti, P. Blanchard, P. Bruzzone, R. Chavan, S. Coda, B.P. Duval, A. Fasoli, B. Labit, B. Lipschultz, T. Lunt, Y. Martin, J.-M. Moret, U. Sheikh, B. Sudki, D. Testa, C. Theiler, M. Toussaint, D. Uglietti, N. Vianello, and M. Wischmeier, Nuc. Mater. Energy Vol. 12, 1106-1111 (2017)

[5] S. Lisgo, M. Istenic, J.M. Canik, R. Buttery, I. Katramados, M. Kotschenreuther, M. Kovari, S. M. Mahajan, M. Shannon, D. Taylor, P. M. Valanju, and the MAST Team, 36th EPS Conference on Plasma Phys. Sofia, June 29 - July 3, 2009 ECA Vol.33E, O-4.046 (2009)

[6] E. Havlíčková, W. Fundamenski, M. Wischmeier, G. Fishpool, and A.W. Morris, Plasma Phys. Control. Fusion 56 (2014) 075008 (19pp)

[7] W.L. Barr \& B.G. Logan, Fus. Tech., 18:2, 251-256 (1990)

[8] H.Y. Guo, C.F. Sang, P.C. Stangeby, L.L. Lao, T.S. Taylor, and D.M. Thomas, Nucl. Fusion 57 (2017) 044001 (5pp).

[9] R.J. Goldston, R. Myers, and J. Schwartz, Phys. Scr. T167 (2016) 014017 (6pp) 
[10] N. Asakura, N. Hosogane, K. Itami, A. Sakasai, S. Sakurai, K. Shimizu, M. Shimada, H. Kubo, S. Higashijma, H. Takenaga et al., J. Nucl. Mater. 266-269, 182 (1999)

[11] ) A. Kallenbach, M. Kaufmann, D.P. Coster, J.C. Fuchs, A. Herrmann, J. Neuhauser, R. Schneider, K. Borrass, H.-S. Bosch, A. Carlson et al., Nucl. Fusion 38, 901 (1999)

[12] B. Lipschultz, B. LaBombard, J. L. Terry, C. Boswell, and I. H. Hutchinson, Fusion Sci. Technol. 51,369 (2007)

[13] H.Y. Guo, H.Q. Wang, J.G. Watkins, L. Casali, B. Covele, A.L. Moser, T. Osborne, C.M. Samuell, M.W. Shafer, P.C. Stangeby et al., Nucl. Fusion 59, 086054 (2019)

[14] A. Kallenbach, R. Dux, V. Mertens, O. Gruber, G. Haas, M. Kaufman, W. Poschenrieder, F. Ryter, H. Zohn, M. Alexander et al., Nucl. Fusion 35, 1231 (1995)

[15] G.F. Matthews, B. Balet, J.G. Cordey, S.J. Davies, G.M. Fishpool, H.Y. Guo, L.D. Horton, M.G. von Hellermann, L.C. Ingesson, J. Lingertat, et al., Nuc. Fus., Vol. 39, No. 1 (2013)

[16] J. Rapp, T. Eich, M. von Hellermann, A. Herrmann, L. C. Ingesson, S. Jachmich, G. F. Matthews, V. Philipps, G. Saibene et al., Plasma Phys. Control. Fusion 44, 639 (2002)

[17] F. Effenberg, S. Brezinsek, Y. Feng, R. König, M. Krychowiak, M. Jakubowski, H. Niemann, V. Perseo, O. Schmitz, D. Zhang et al., Nucl. Fusion 59, 106020 (2019)

[18] M. Sakamoto, K. Oki, Y. Nakashima, Y. Akabane, Y. Nagatsuka, M. Yoshikawa, R. Nohara, K. Hosoi, H. Takeda, K. Ichimura, J. Kohagura, M. Yoshikawa, M. Ichimura, and T. Imai, Fus. Science Tech., 63:1T, 188-192 (2013)

[19] V. A. Soukhanovskii, Plasma Phys. Control. Fusion 59 (2017) 064005 (12pp)

[20] N. Asakura, , K. Hoshino, S. Suzuki, S. Tokunaga, Y. Someya, H. Utoh, H. Kudo, Y. Sakamoto, R. Hiwatari, K. Tobita, K. Shimizu, K. Ezato, Y. Seki, N. Ohno, Y. Ueda, and Joint Special Team for DEMO Design, 2017 Nucl. Fusion 57126050

[21] H.J.N. van Eck, G.R.A. Akkermans, S. Alonso van der Westen, D.U.B. Aussems, M. van Berkel, S. Brons, I.G.J. Classen, H.J. van der Meiden, T.W. Morgan, M.J. van de Pol, et al., Fus. Eng. and Design, 142, 26-32 (2019).

[22] H.J. van der Meiden, A.R. Lof, M.A. van den Berg, S. Brons, A.J.H. Donné, H.J.N. van Eck, P. Koelman, W.R. Koppers, O.G. Kruijt, N.N. Naumenko, et al., Rev. Sci. Instrum. 83 (2012) 123505, https://doi.org/10.1063/1.4768527.

[23] G.R.A. Akkermans, I.G.J. Classen, R. Perillo, H.J. van der Meiden, F. Federici, and S. Brezinsek, Phys. Plasmas 27, 102509 (2020)

[24] A. Matsubara, T. Watanabe, T. Sugimoto, S. Sudo, and K. Sato, J. Plasma Fusion Res. SERIES, Vol. 6 (2004) 433-436

[25] P.C. Stangeby, The Plasma Boundary of Magnetic Fusion Devices, Taylor \& Francis Group, 2000.

[26] J. A. M. van der Mullen, Excitation equilibria in plasmas; a classification, Physics Reports Vol. 191, pp 109-220, 1990.

[27] The iso-nuclear master files from the ADF11 class on the open version of the atomic data and analysis structure, http://open.adas.ac.uk/, version 2.0, OPEN-ADAS . 


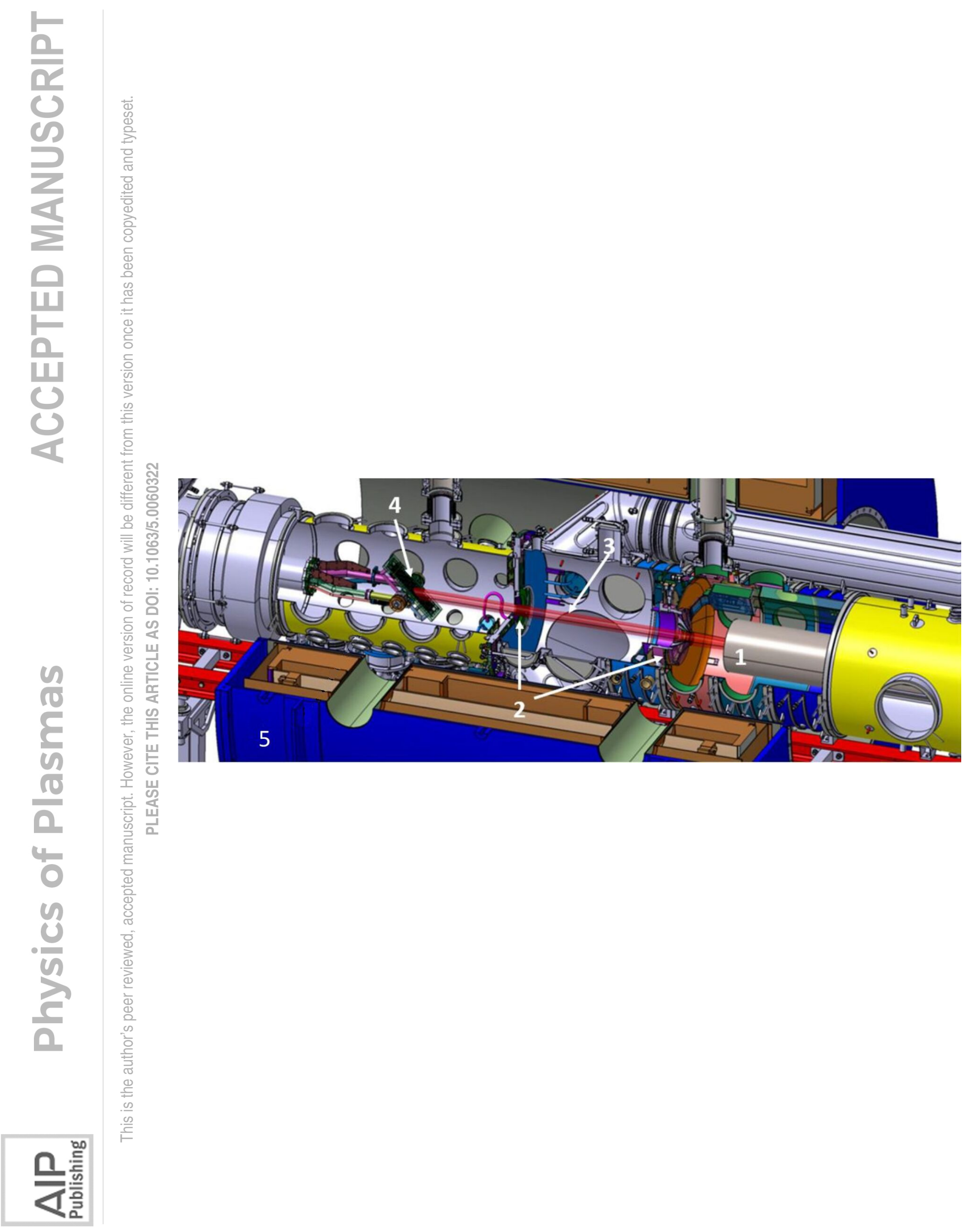




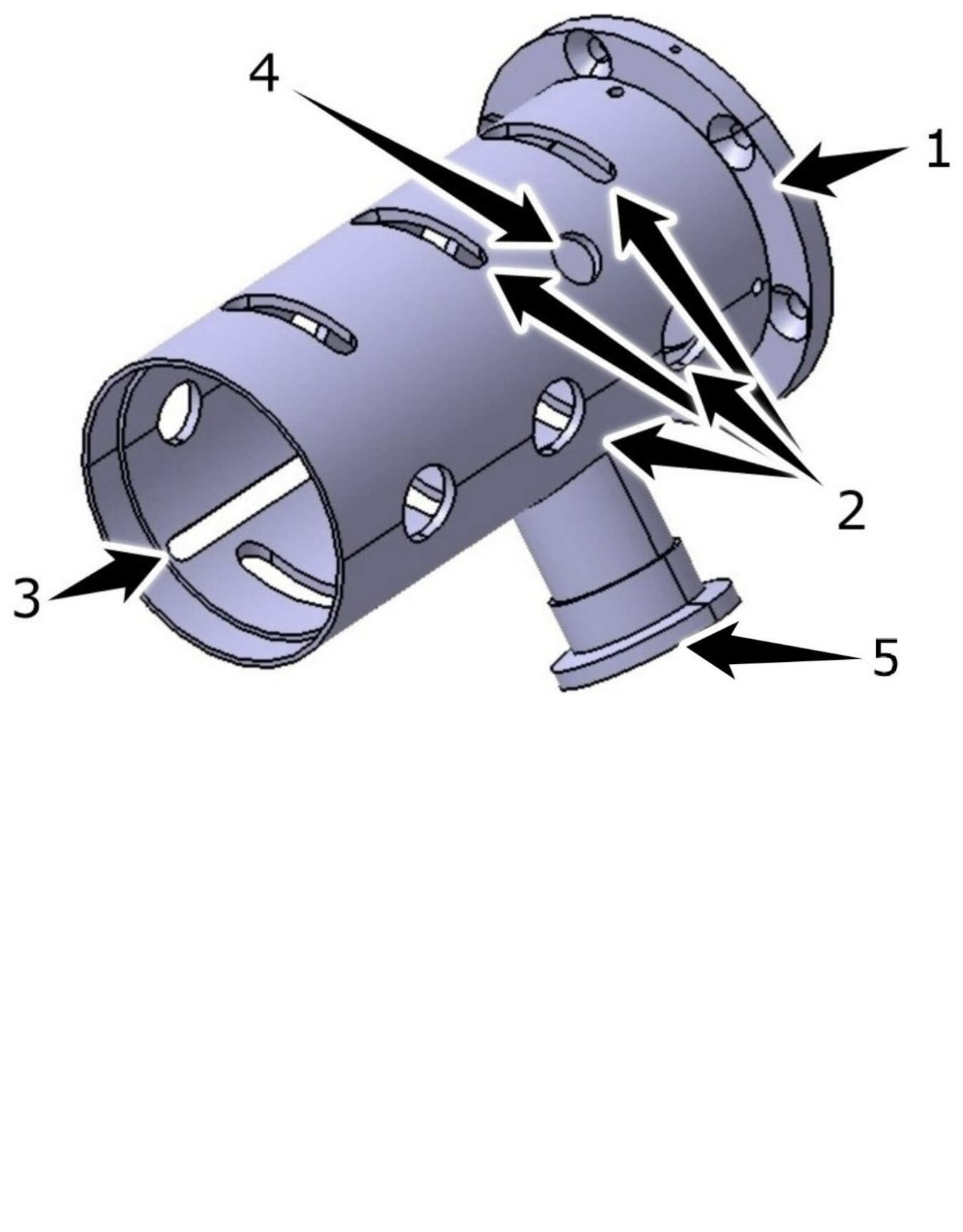



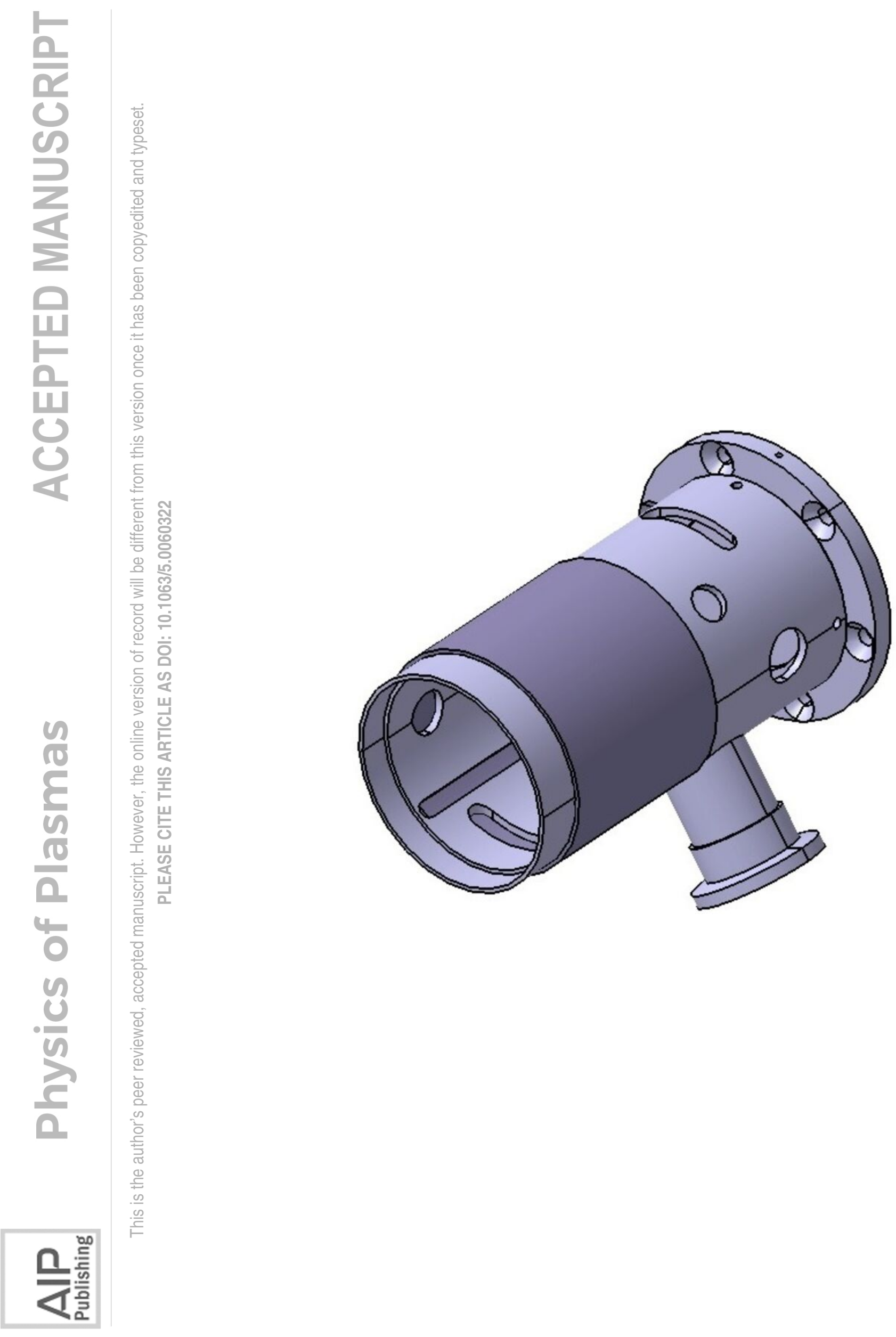

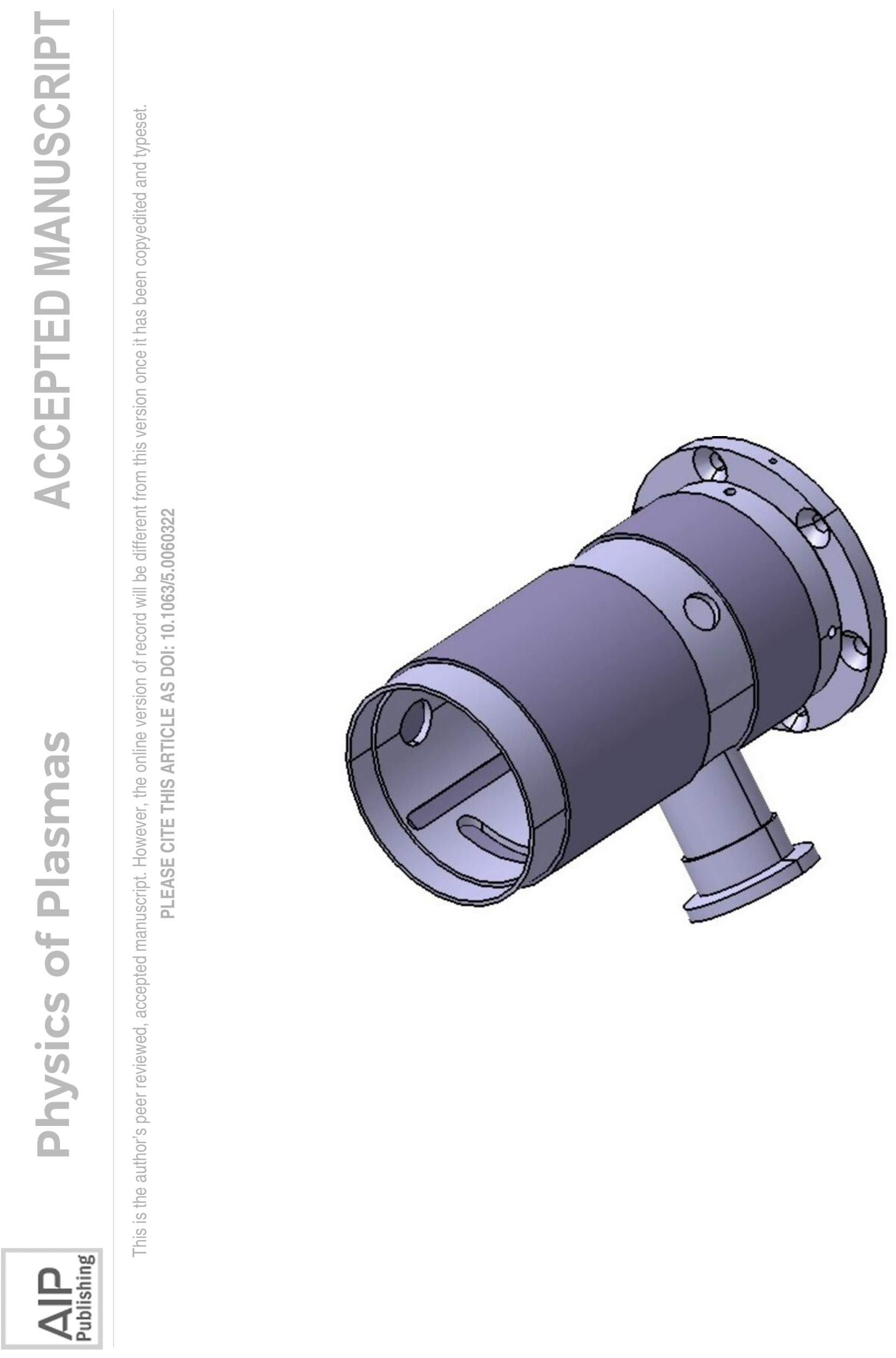


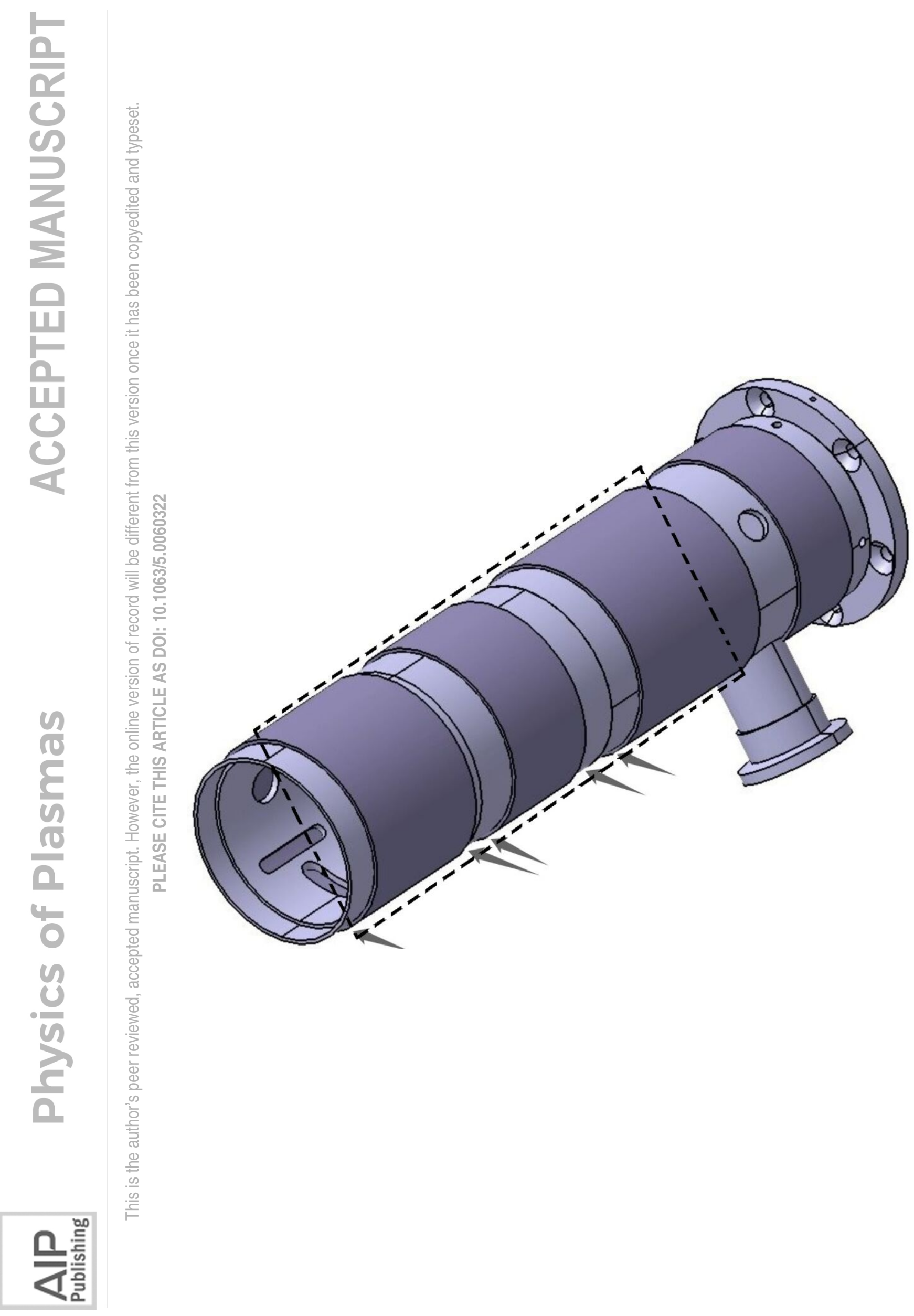


a)

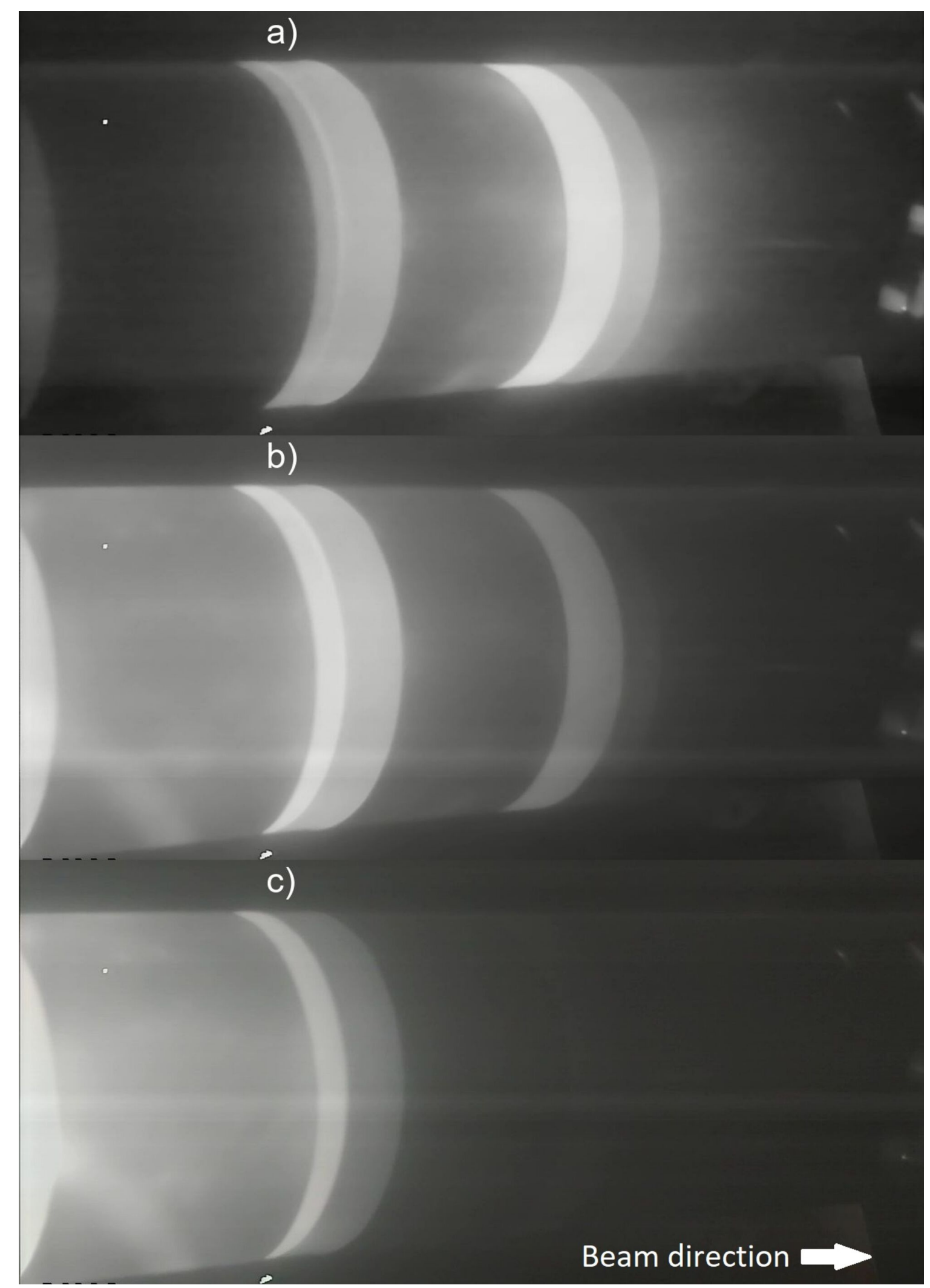

는 


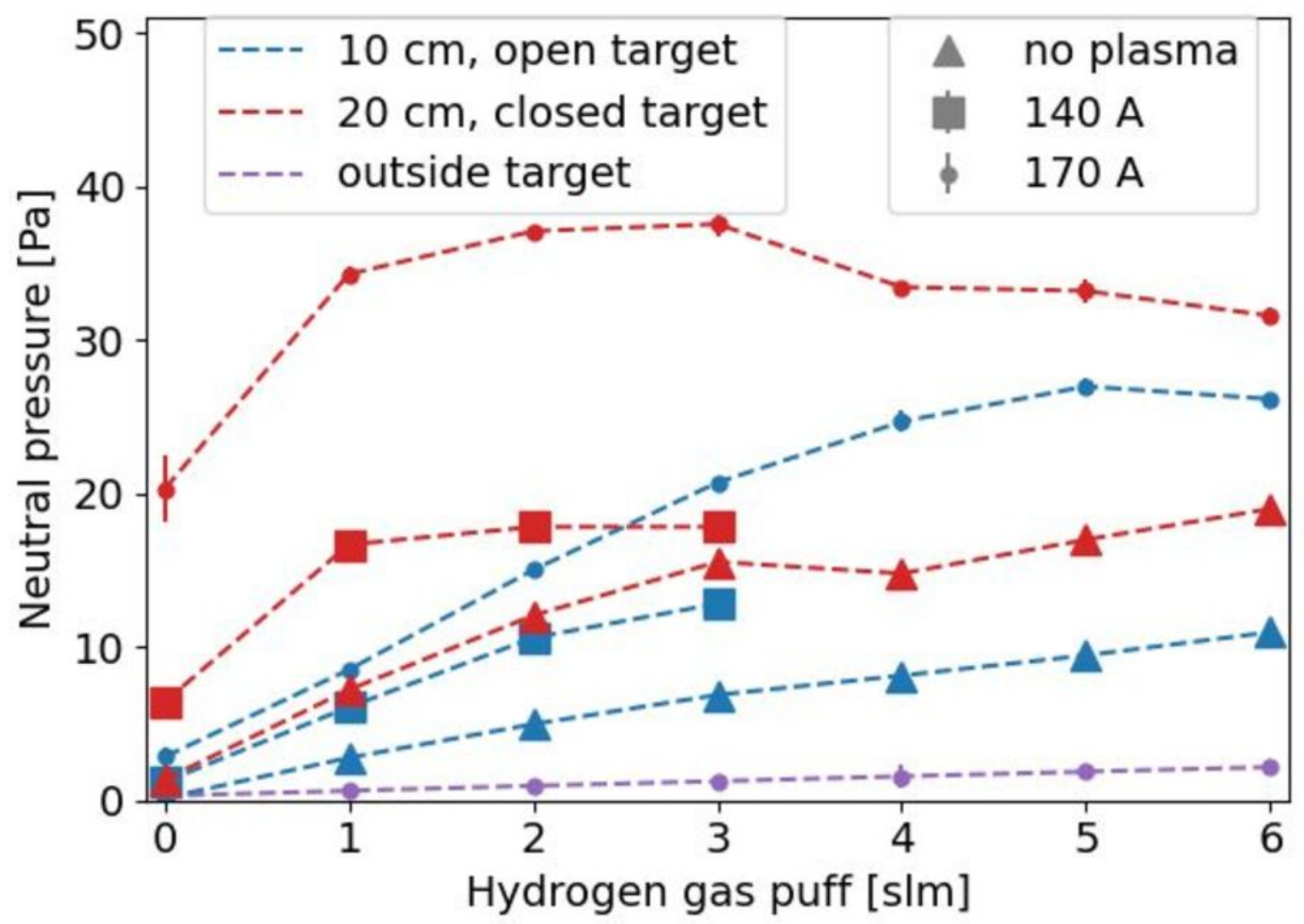

兄耪言 

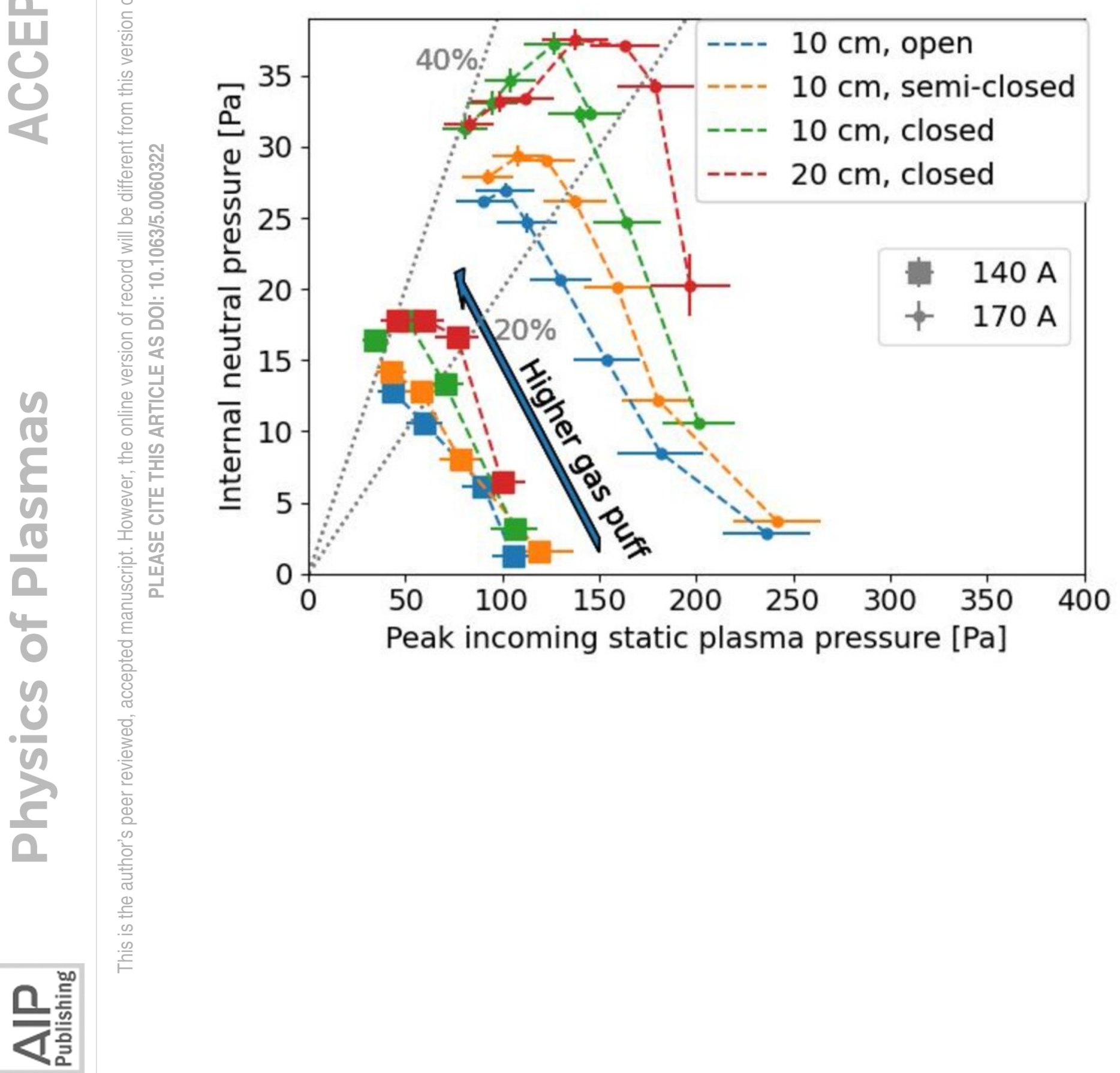


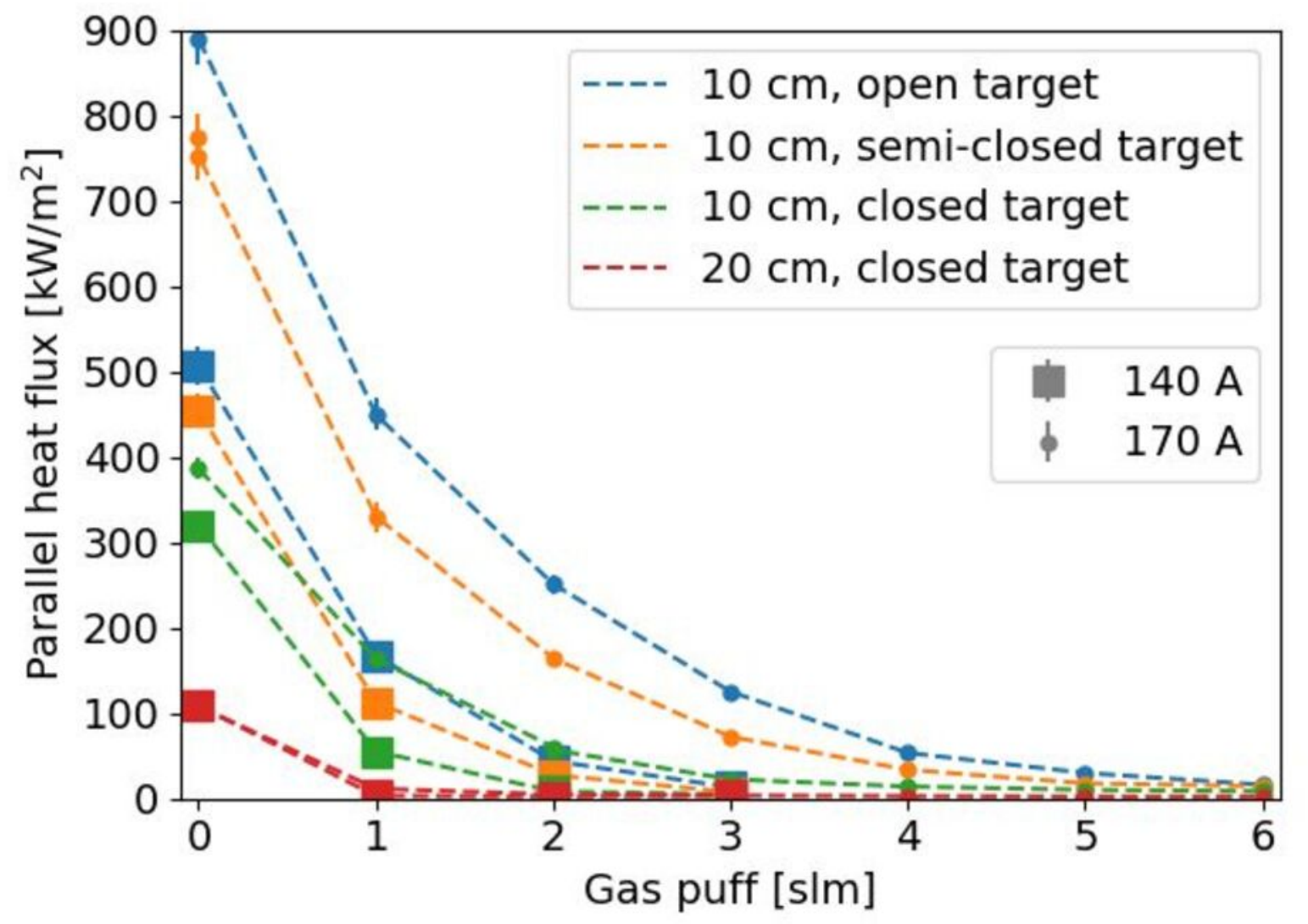




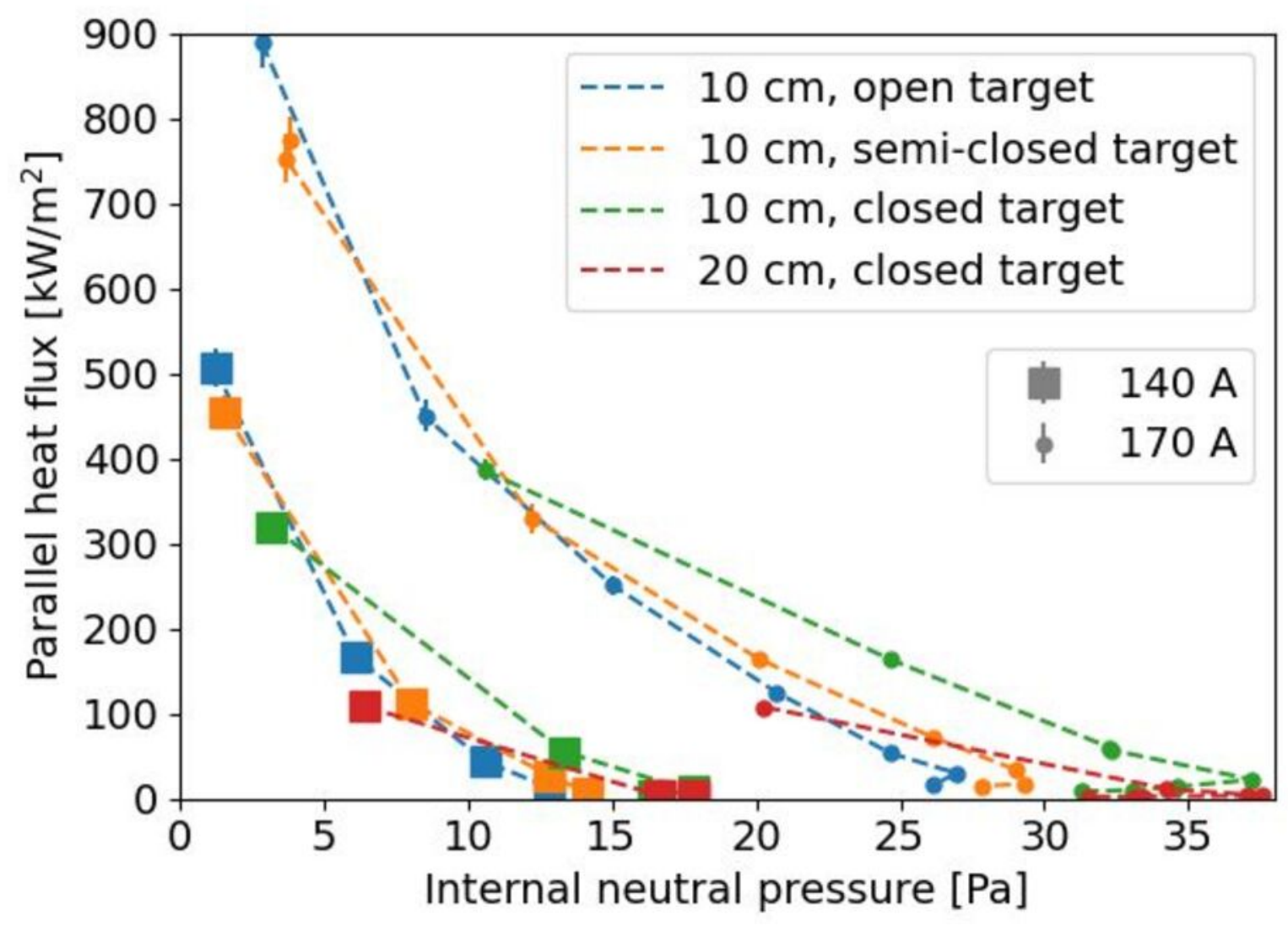




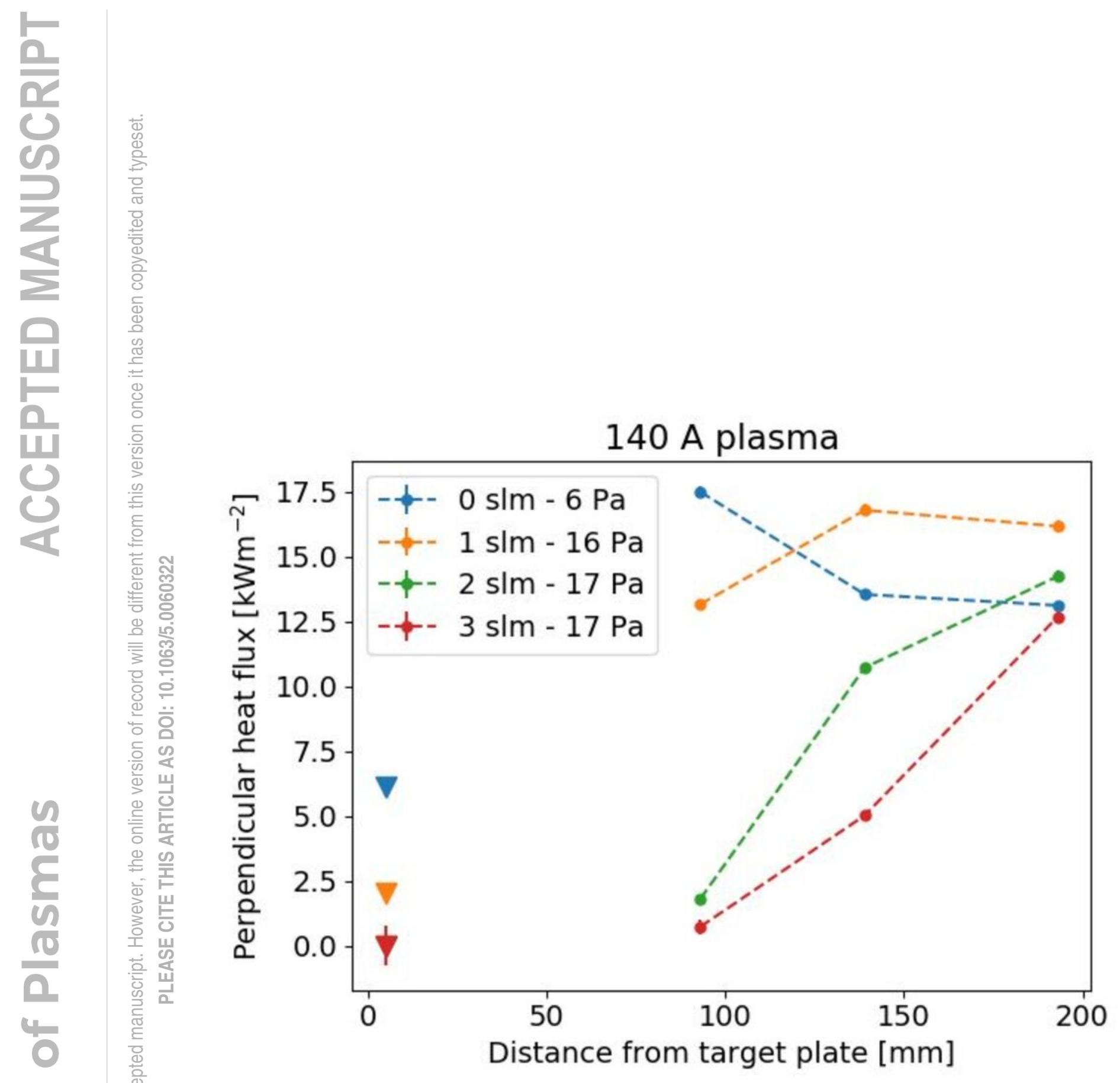


170 A plasma

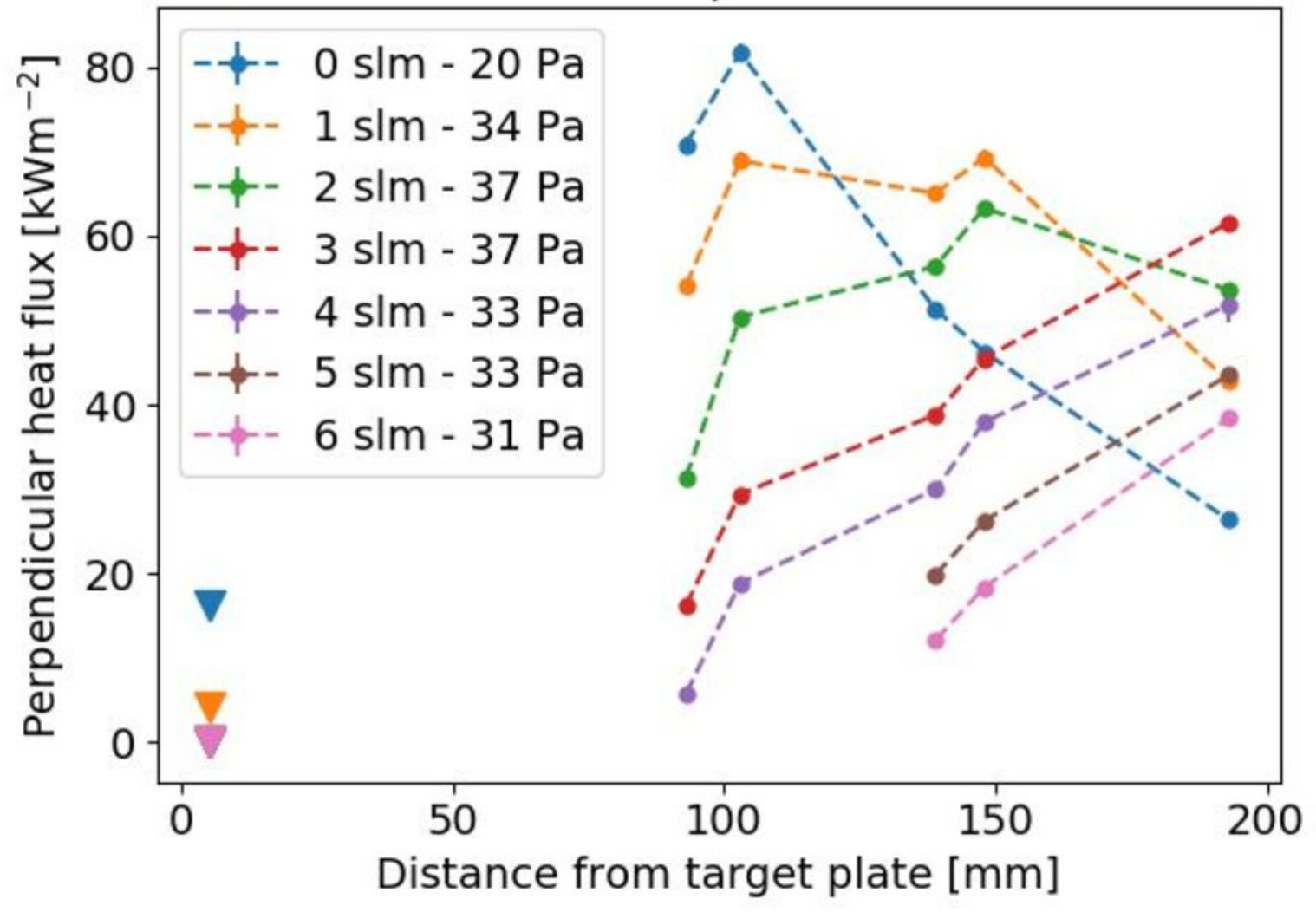




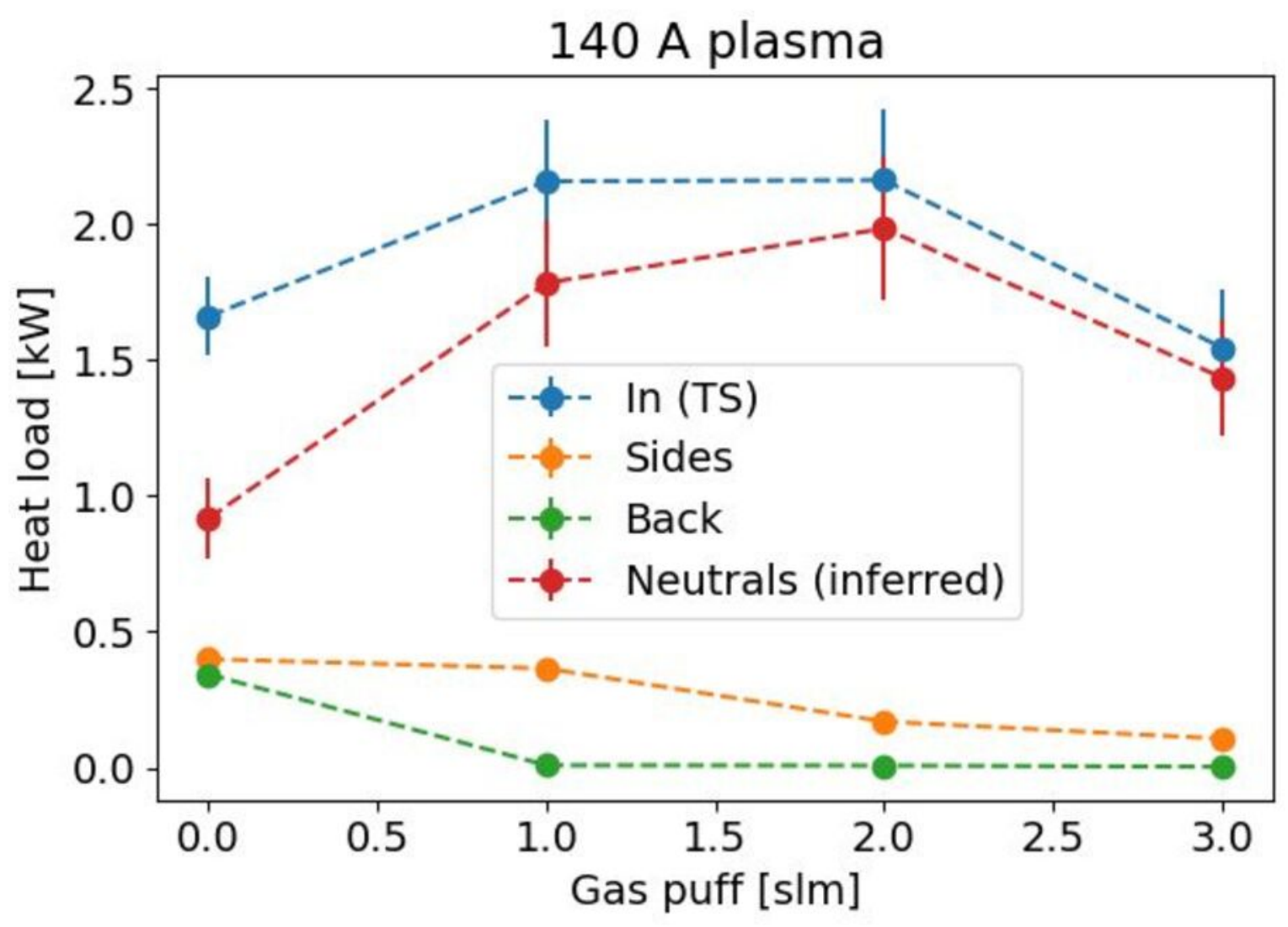

는 


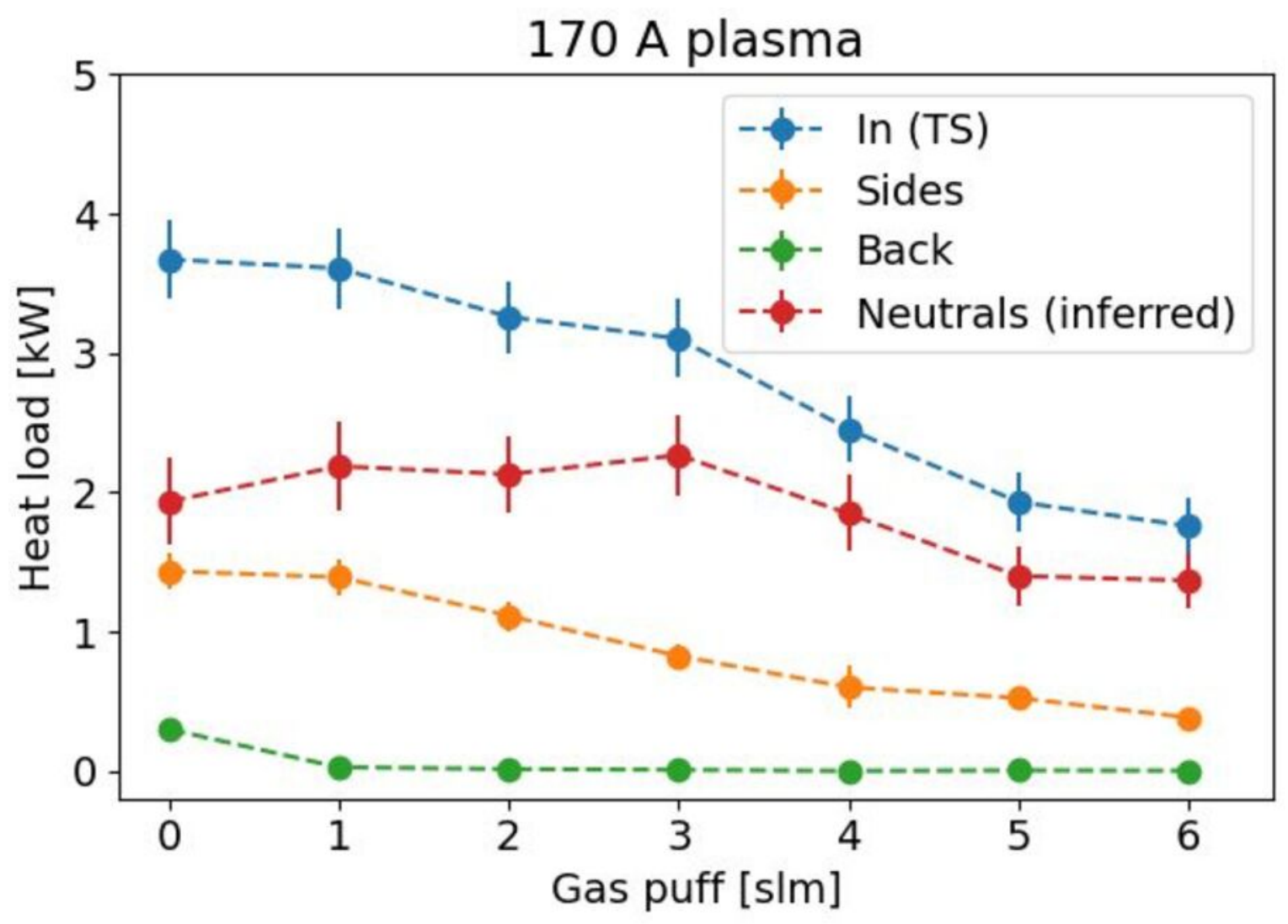




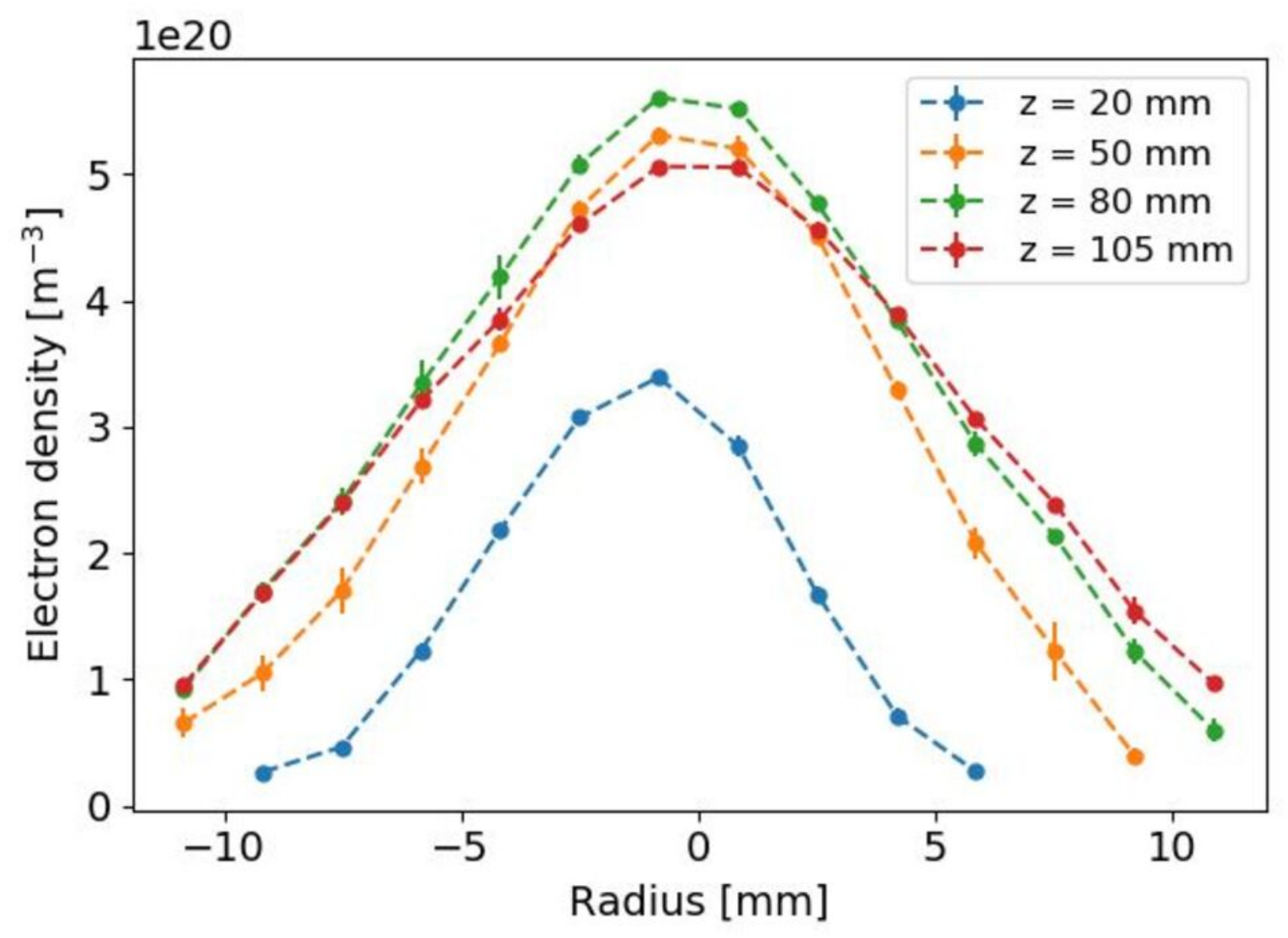

兄旁言 


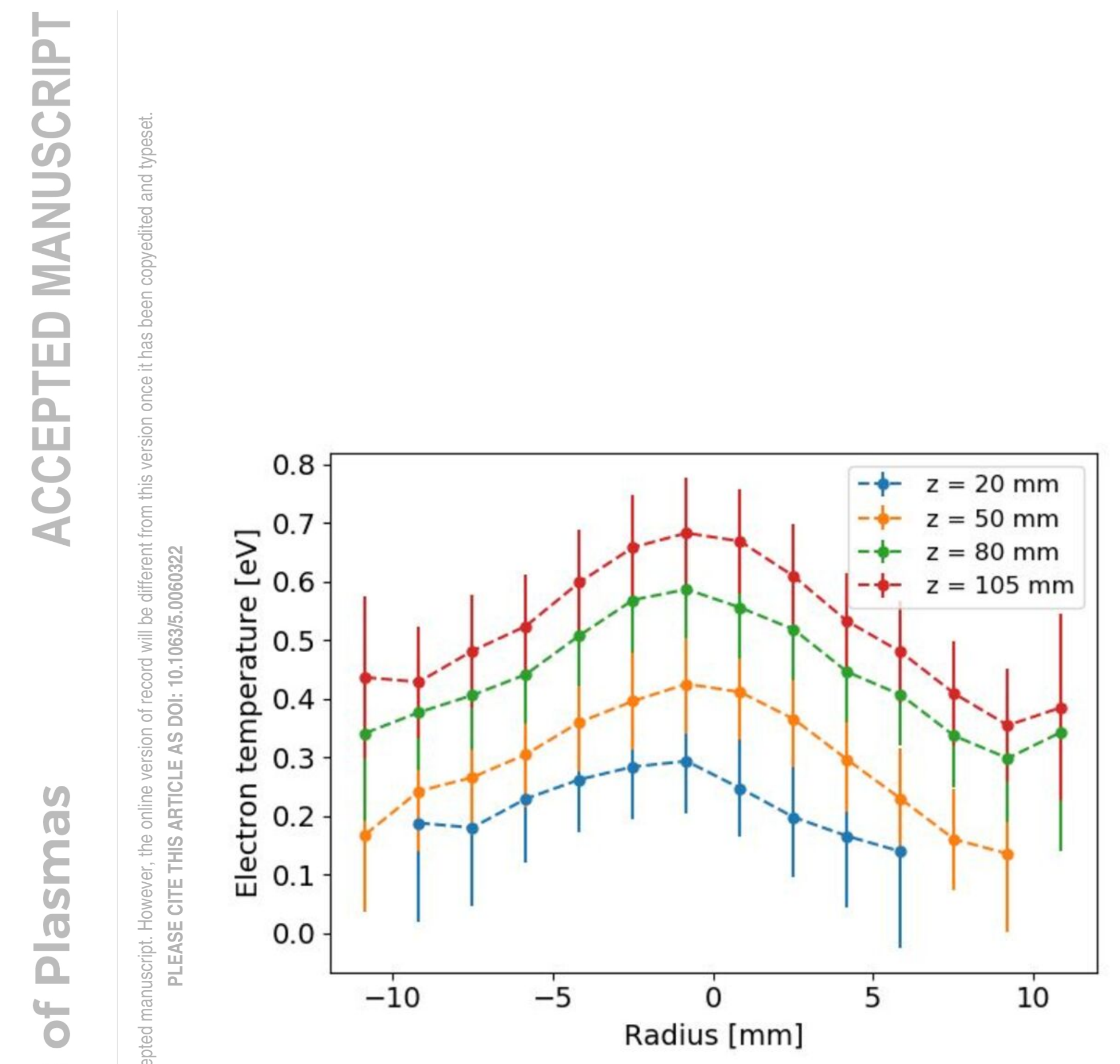




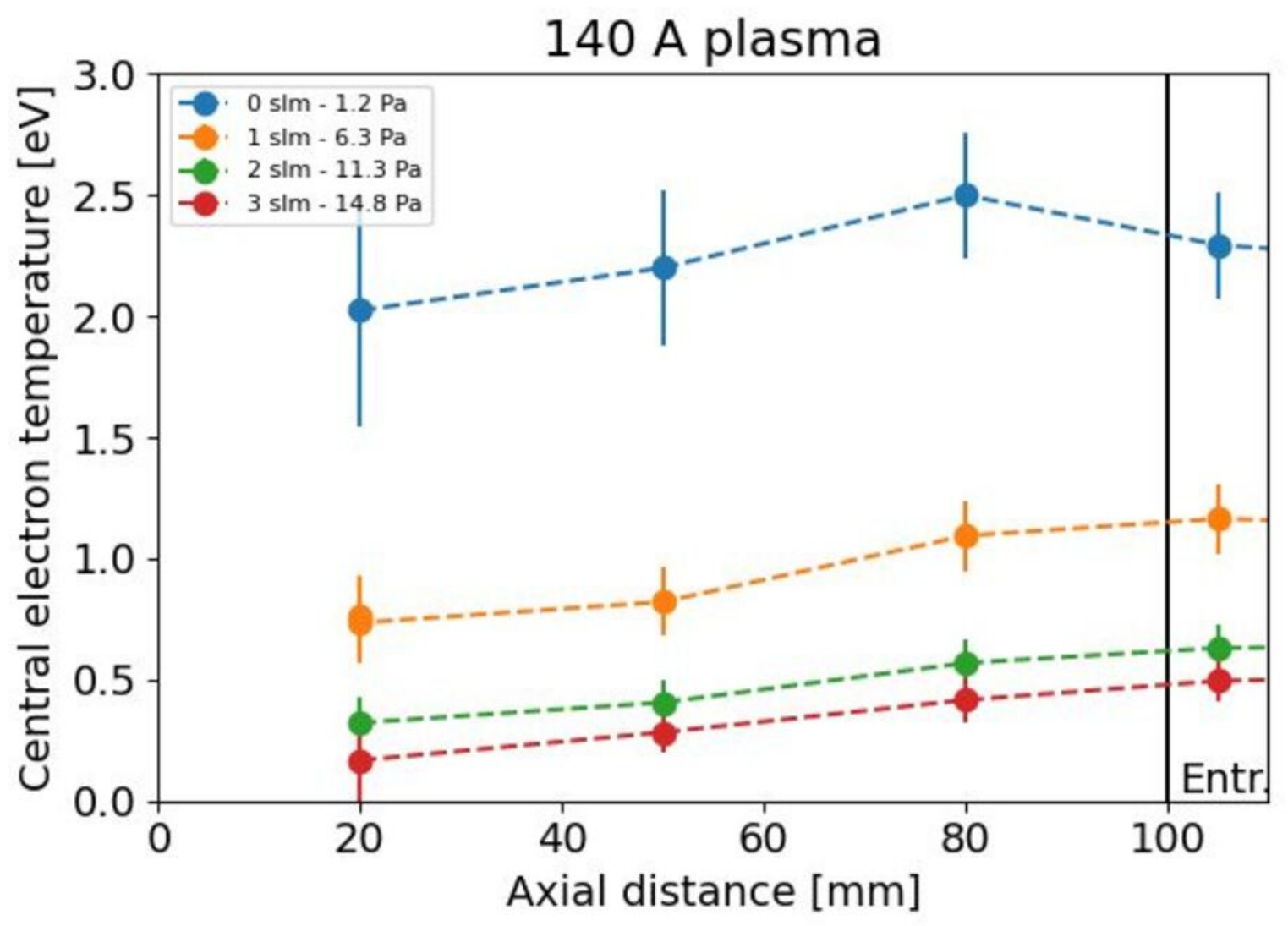


170 A plasma

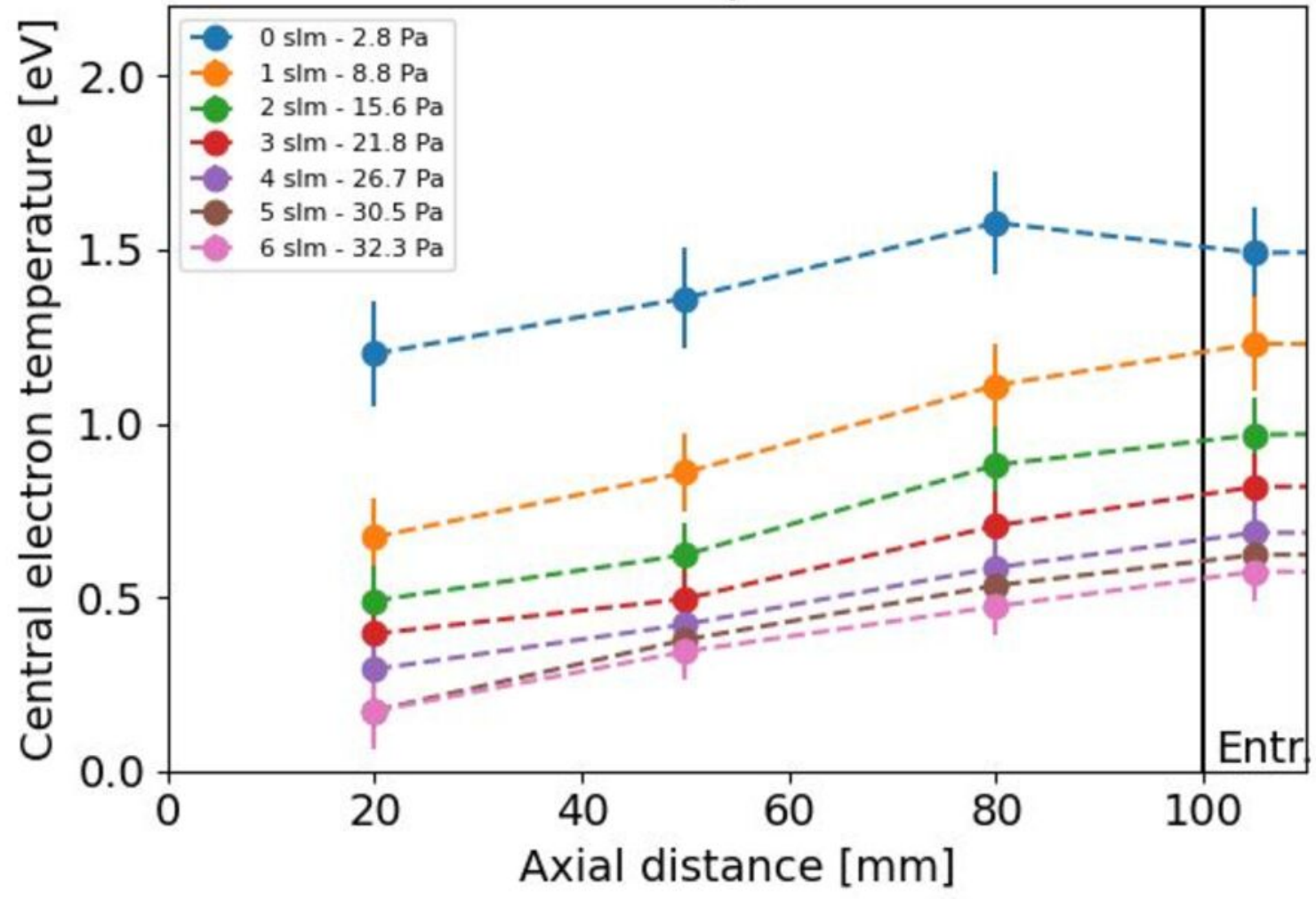




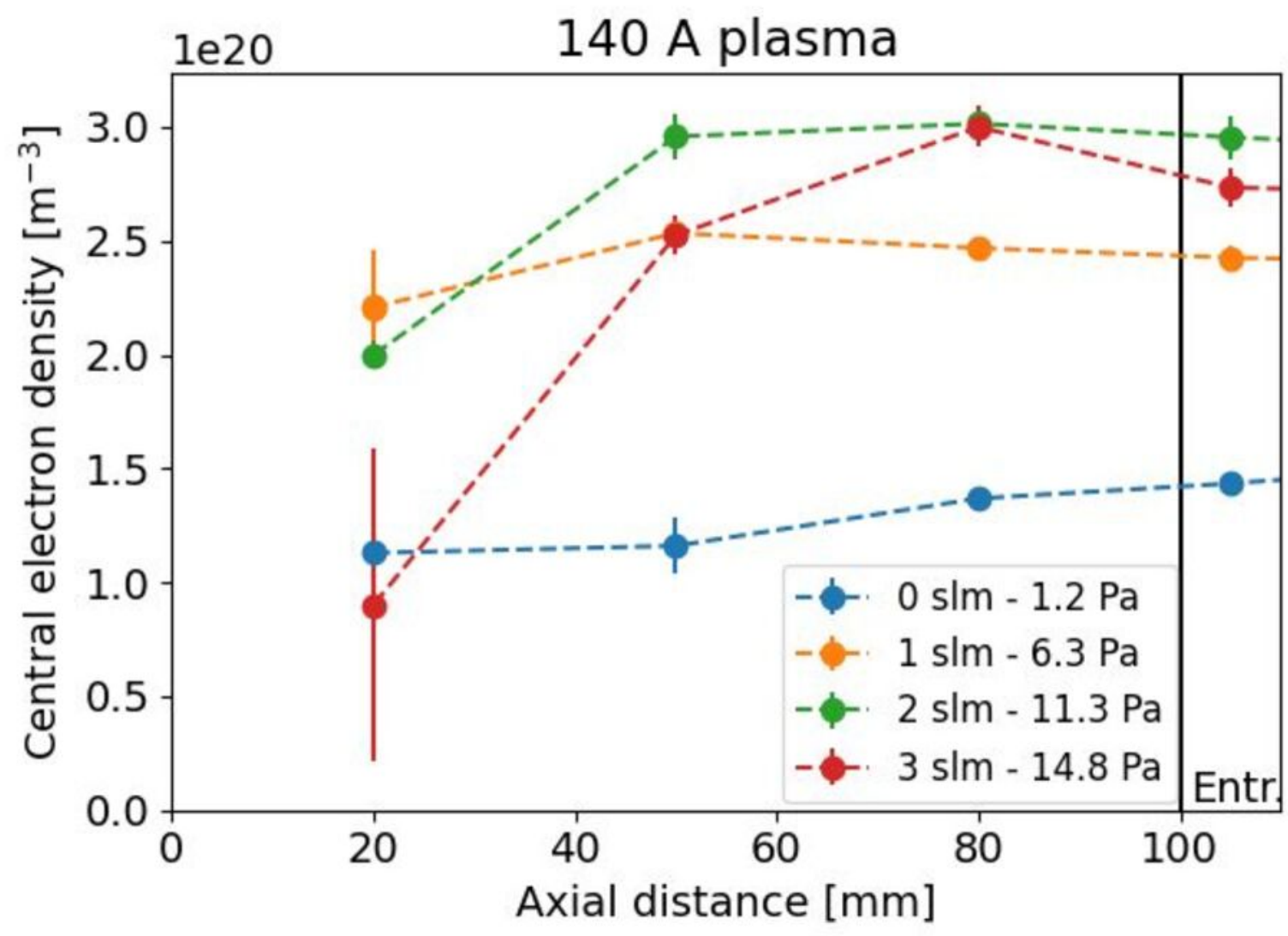




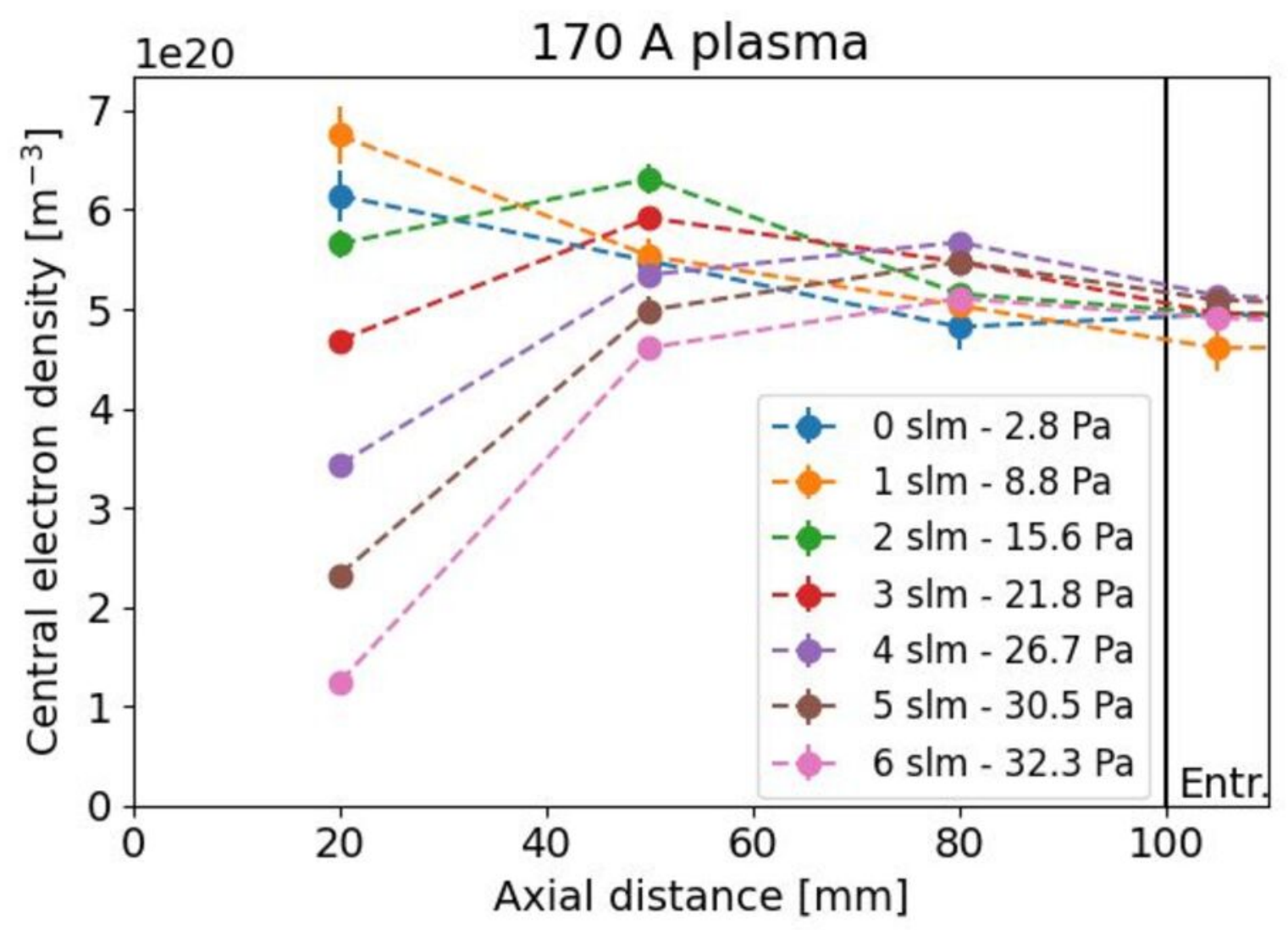



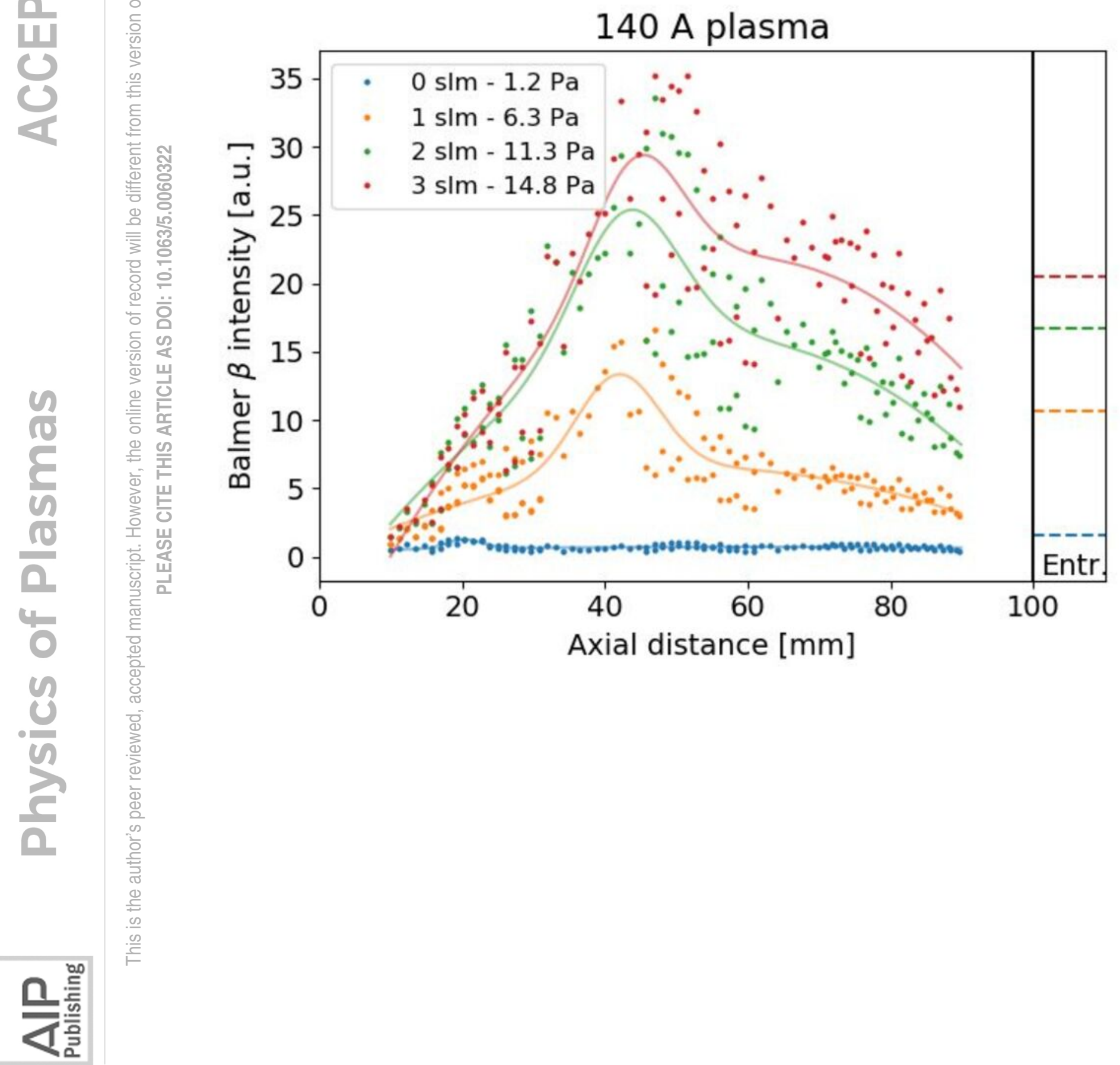

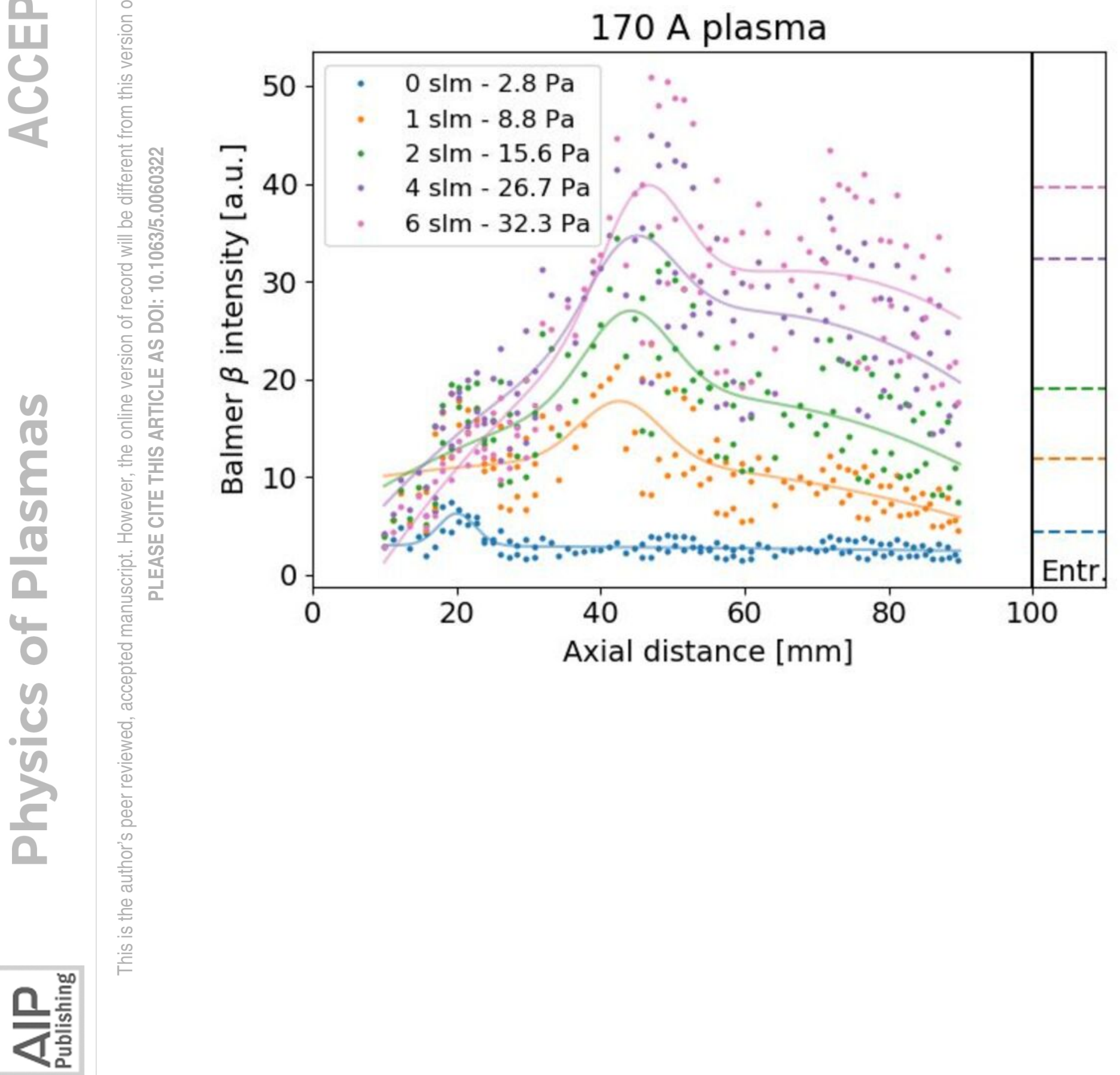


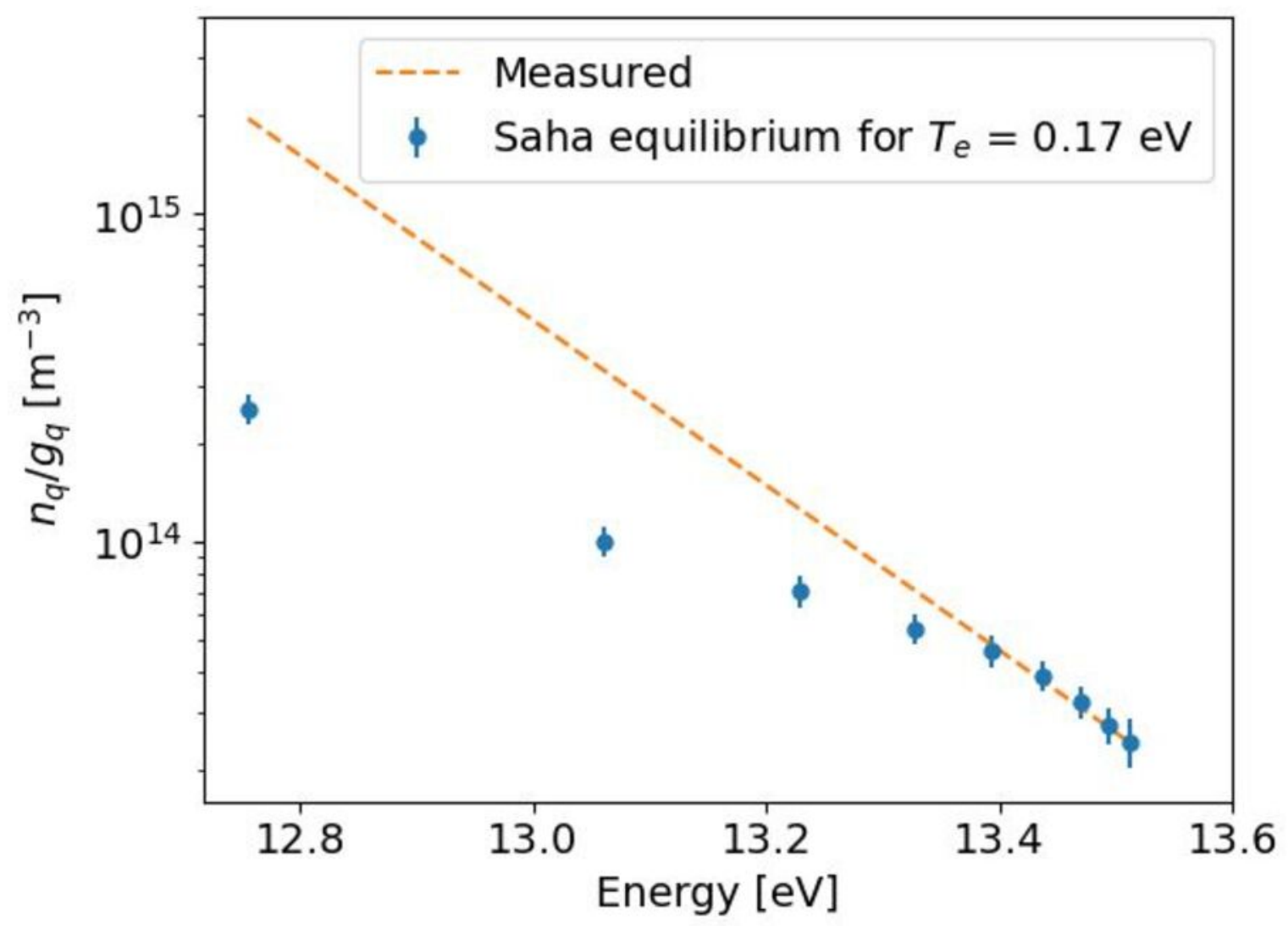

